\title{
Olive Extract Ameliorates Oxidative Stress and Inflammation, and Protects Intestinal Villus and Microbiota in Piglets Induced by Lipopolysaccharides
}

\section{Yu Zhang}

Zhejiang University

\section{Zhao-Xi Deng}

Zhejiang University

Mao-Long He

Lucta SA

Xin M. Luo

Virginia Polytechnic Institute and State University

Jian-Xin Liu

Zhejiang University

Haifeng Wang ( $\square$ haifengwang@zju.edu.cn )

Zhejiang University https://orcid.org/0000-0002-8211-9691

\section{Research}

Keywords: Olive extract, Anti-oxidative, Anti-inflammatory, Intestinal morphology, Piglet

Posted Date: November 5th, 2020

DOl: https://doi.org/10.21203/rs.3.rs-101171/v1

License: (c) (i) This work is licensed under a Creative Commons Attribution 4.0 International License.

Read Full License 


\section{Abstract}

Background: The olive extract contains compounds with antioxidant and anti-inflammatory properties. This study was designed to investigate whether olive cake extract, enriched with maslinic acid and hydroxytyrosol, alleviates the lipopolysaccharides (LPS)-induced oxidative stress, inflammation and intestinal villus damage in piglets.

Methods: Thirty weaned piglets $(6.9 \pm 0.9 \mathrm{~kg})$ were assigned to five groups using a randomized complete block design. Piglets were fed a basal diet before intraperitoneal (i.p.) injection of physiological saline (C); fed a basal diet alone (CL) or fed a basal diet plus olive extract $(\mathrm{OL})$, antibiotics (AL), or olive extract and antibiotics (OAL) before i.p. injection of LPS. The feeding lasted for 2 weeks. Piglets were euthanized $4 \mathrm{~h}$ after LPS injection. Systemic anti-oxidant and inflammation levels were measured and villus morphology in the intestine was examined.

Results: Compared with those in the $\mathrm{C}$ group, piglets in the $\mathrm{CL}$ group had significantly lower GSH-Px, SOD, ALB levels and higher MDA, NO, LDH, ALT and AST levels in the serum $(P<0.05)$. Compared with the CL group, piglets in OL, AL, and OAL groups had significantly higher serum GSH-Px, SOD and ALB levels and lower MDA, NO, LDH, ALT and AST levels $(P<0.05)$. LPS administration significantly increased the serum concentration of TNF-a, IL-6, DAO and D-xylose in the CL group compared with the control group $(P<0.05)$. Piglets in OL, AL, and OAL groups had significantly lower serum TNF-a, IL-6, DAO and D-xylose levels and higher IL-10 level $(P<0.05)$. In the duodenum and ileum of piglets, LPS challenge led to significantly lower villus height $(\mathrm{VH})$, higher crypt depth (CD) and lower VH/CD compared with the control group $(P<0.05)$, whereas, $\mathrm{OL}, \mathrm{AL}$, and $\mathrm{OAL}$ groups had significantly lower $\mathrm{CD}$ and higher $\mathrm{VH} / \mathrm{CD}$ compared with the $\mathrm{CL}$ group $(P<0.05)$. Dietary inclusion of olive extract increased the relative abundance of intestinal Lactobacillus and Clostridium at genus level.

Conclusion: Dietary supplementation with olive extract maslinic acid and hydroxytyrosol improved antioxidative and anti-inflammatory capacity, intestinal structure morphology, and increased the abundance of beneficial intestinal bacteria in weaned piglets challenged by LPS.

\section{Background}

Modern livestock production has become highly intensive and large scaled to increase production efficiency. However, this production environment could add stressors affecting the health and growth of animals(1). Oxidative stress occurs when there is an imbalance between free radical production and antioxidant capacity(2). Weaned piglets are subjected to lots of stress, including changes in nutrition, separation from their mothers and littermates, and a new environment, which cause reduced growth and compromised small intestine function(3). In piglets, weaning disrupts the oxidative balance, cause oxidative stress and leads to free radical-mediated oxidative injury (4). The maintenance of redox homeostasis, which is very important for normal cellular processes and organic function, highly depends on the balance between pro-oxidative and anti-oxidative systems(5). The intestinal tract is the primary 
location of the digestion and absorption of nutrients in the animal body and it acts as the largest immune organ(6). The intestinal tract is the first line of defense against bacterial and harmful endogenous and exogenous substances(7). Oxidative stress induces intestinal cell cycle arrest and apoptosis(8) and decreases the expression of intestinal tight junction proteins and mucosal barrier function(9). Together this results in the deterioration of gastrointestinal digestive and absorptive functions in piglets(5).

The Olive tree (Olea europaea L.), a native of the Mediterranean basin and parts of Asia, is widely cultivated in many other parts of the world for the production of olive oil and table olives. Olive fruit contains an appreciable concentration, $1-3 \%$ of fresh pulp weight, of hydrophilic and lipophilic (cresols) phenolic compounds that possess antioxidant, anticarcinogenic, anti-inflammatory, antimicrobial, and antihypertensive biological activities(10). The phenolic compounds in olives have been described as oleuropein, hydroxytyrosol and verbascoside(11). Previous studies have shown that olives contain substances similar to ibuprofen, anon-steroidal anti-inflammatory drug with anti-inflammatory and antioxidant functions(12). The Mediterranean diet has attracted worldwide attention, mainly because it can reduce the incidence of cardiovascular disease and cancer on the premise of meeting human dietary needs(13). Olive leaves have been used as folk medicines to remedy diseases including fever and malaria(14). Olive leaf extract, containing polyphenols such as oleuropein and hydroxytyrosol, reverses the chronic inflammation and oxidative stress observed in the rat model of diet-induced obesity(15). Although olive cake is an economical biomass present in large quantities, it causes some environmental problems for Mediterranean countries(16). Olive cake is considered a rich source of phenolic compounds with a wide array of biological activities. Antioxidant attributes have been investigated in olive cakes for anti-radical activities(17). The recovery of phenolic compounds from olive cake has a potential use in the pharmaceutical and nutraceutical industries.

In this study, induced inflammation in piglets by challenging them with LPS(18), and investigated the protective effect of olive extract supplementation on lipopolysaccharide (LPS) induced-damage to the intestine of piglets. We hypothesize that dietary inclusion of olive extract maslinic acid and hydroxytyrosol would enhance antioxidant and anti-inflammatory capacity in weaned piglets and improve the intestinal health and performance of weaning piglets. Therefore, this study was designed to examine the effects of olive extract addition on intestinal function and growth performance in piglets.

\section{Methods}

\section{Animal and diets}

Thirty piglets (Duroc $\times$ Landrace $\times$ Large White, including males and female, $6.9 \pm 0.9 \mathrm{~kg}$ ) aged $28 \pm 1 \mathrm{~d}$ were assigned to five groups ( $n=6 /$ group). All animal procedures were performed in full accordance with the Regulation for the Use of Experimental Animals in Zhejiang Province, China. This work was specifically approved by the Animal Care and Use Committee of Zhejiang University (ethics code permit no. ZJU20170529). Piglets were fed a basal diet before intraperitoneal (i.p.) injection of physiological saline (C). Piglets in the other four groups were fed the basal diet alone (CL) or fed a basal diet plus olive 
extract (OL), antibiotics (AL), or olive cake extract and antibiotics (OAL) before i.p. injection of LPS $(20 \mu \mathrm{g} / \mathrm{kg}$ body weight, Sigma-Aldrich, Saint Louis, MO, USA). The $0.1 \%$ olive cake extract in diet mainly included maslinic acid and hydroxytyrosol. Antibiotics included $120 \mathrm{mg} / \mathrm{kg}$ oxytetracycline-calcium and $16 \mathrm{mg} / \mathrm{kg}$ enduracidin in diet. The basal feed composition is shown in Table 1. 
Table 1

Basal diet ingredients and chemical composition.

\section{Items, $\mathbf{g} / \mathbf{k g}$}

Ingredients

Corn

Soybean meal

Fermented soybean meal

Wheat flour

Soybean protein concentrate

Soybean oil

14.4

Fish meal 20

Whey powder 20

Brewer's yeast 10

Sucrose 20

Glucose 20

Calcium hydrophosphate 10

Limestone 7

Sodium chloride 3

L-Lysine hydrochloride 1.8

DL-Methionine 0.8

Vitamin-mineral premix ${ }^{*}$ 5

Nutrition composition

Digestible energy ${ }^{\dagger}, \mathrm{MJ} / \mathrm{kg}$ (calculated)

141.48

Crude protein

183.62

Calcium

8.52

*Provided per kilogram of diet: retinyl acetate, 25,000 IU; cholecalciferol, 3,750 IU; rac-a-tocopheryl acetate, $30 \mathrm{lU}$; menadione, $3 \mathrm{mg}$; thiamin, $5 \mathrm{mg}$; riboflavin, $10 \mathrm{mg}$; pantothenic acid, $40 \mathrm{mg}$; niacin, $50 \mathrm{mg}$; pyridoxol, $8 \mathrm{mg}$; biotin, $0.3 \mathrm{mg}$; folic acid, $3 \mathrm{mg}$; cobyrinic acid, $0.6 \mathrm{mg} ; \mathrm{Zn}, 250 \mathrm{mg} ; \mathrm{Fe}, 300 \mathrm{mg}$; $\mathrm{Cu}, 400 \mathrm{mg} ; \mathrm{Mn}, 80 \mathrm{mg} ; \mathrm{l}, 0.5 \mathrm{mg}$; Se, $0.6 \mathrm{mg}$.

${ }^{\dagger}$ Digestible energy was calculated. 


\section{Items, $\mathbf{g} / \mathbf{k g}$}

Lysine

13.04

Methionine

3.61

*Provided per kilogram of diet: retinyl acetate, 25,000 IU; cholecalciferol, 3,750 IU; rac-a-tocopheryl acetate, $30 \mathrm{lU}$; menadione, $3 \mathrm{mg}$; thiamin, $5 \mathrm{mg}$; riboflavin, $10 \mathrm{mg}$; pantothenic acid, $40 \mathrm{mg}$; niacin, $50 \mathrm{mg}$; pyridoxol, $8 \mathrm{mg}$; biotin, $0.3 \mathrm{mg}$; folic acid, $3 \mathrm{mg}$; cobyrinic acid, $0.6 \mathrm{mg} ; \mathrm{Zn}, 250 \mathrm{mg} ; \mathrm{Fe}, 300 \mathrm{mg}$; $\mathrm{Cu}, 400 \mathrm{mg} ; \mathrm{Mn}, 80 \mathrm{mg} ; \mathrm{l}, 0.5 \mathrm{mg} ; \mathrm{Se}, 0.6 \mathrm{mg}$.

${ }^{\dagger}$ Digestible energy was calculated.

Animals were allowed free access to water and feed. The feeding lasted two weeks. Four hours after LPS challenge, piglets were euthanized via an intravenous injection of sodium pentobarbital $(50 \mathrm{mg} / \mathrm{kg}$ body weight)(19). Blood was collected and centrifuged at $4000 \times \mathrm{g}$ for $10 \mathrm{~min}$ to collect serum. Serum samples were stored at $-80^{\circ} \mathrm{C}$ for later analysis. Segments $(1 \mathrm{~cm} \times 1 \mathrm{~cm})$ of the duodenum, jejunum and ileum were gently flushed with $5 \mathrm{~mL} 0.9 \%$ saline twice, and then fixed in $10 \%$ formalin fixative solution for use in histopathological(20). The luminal contents of the ileum were collected, frozen with liquid nitrogen and stored at $-80^{\circ} \mathrm{C}$ for further analysis.

\section{Serum Analysis}

Serum glucose (Glu; F006-1-1), total protein (TP; A045-1-1), cholesterol (T-CHO; A111-1) and malondialdehyde (MDA; A003-1) level, and activity of glutathione peroxidase (GSH-Px; A005), nitric oxide (NO; A013-2), superoxide dismutase (SOD; A001-3), activities of alanine aminotransferase (ALT; C009), aspartate aminotransferase (AST; C010), lactate dehydrogenase (LDH; A020-2), albumin (ALB; A028-2-1), diamine oxidase (DAO; A088) activity, and D-xylose (A035) concentration in the serum were determined by kinetics-based assays with commercially available kits (Nanjing Jiancheng Bioengineering Institution, Nanjing, China) using an automatic biochemistry analyzer (SELECTA XL; Vital Scientific) following the manufacturer's protocol. Serum TNF-a, IL-6, and IL-10 level were measured by enzyme-linked immunosorbent assay (ELISA) using commercially available kits (No. H052, No. H007, and No. H009 for TNF-a, IL-6 and IL-10, respectively; Nanjing Jiancheng Bioengineering Institution, Nanjing, China).

\section{Gastrointestinal Ph Value And Organ Weight}

Rectal temperature and gastrointestinal pH value were determined in stomach, duodenum, jejunum, ileum, and colon. Spleen weight was determined.

\section{Histomorphological Analysis}


In brief, the intestine segments fixed in $10 \%$ formalin solution were dehydrated with different alcohol concentrations, and soaked in paraffin as previously described(21). Cross-sections were cut into 5-8 micron thin slices, stained with hematoxylin and eosin(22). Histological assays were performed using Scion image software (Scion Image; Scion Corporation, Frederick, MD) to determine villus height and crypt depth in duodenum, jejunum, and ileum sections.

\section{Sequencing Of Bacterial 16s Rrna In Feces}

The microbial community in the pig intestine was detected and analyzed using high-throughput sequencing. Primers 515F (5'-ACTCCTACGGGAGGCAGCA-3') and 806R (5'-GGACTACHVGGGTWTCTAAT$\left.3^{\prime}\right)$ were used to amplify the V4 variable region of the $16 \mathrm{~S}$ rDNA gene. The constructed amplicon library was sequenced using the Illumina Hiseq2500 platform (Guangdong Magigene Biotechnology Co., Ltd. Guangdong, China). Paired sequence reads were assembled using Flash (V1.2.11, http://ccb.jhu.edu/software/FLASH/). The default QIIME (1.9.1) was used for quality filtering(23). The trimmed sequence was chimeric filtered, single sequences is discarded, and the obtained sequences were allocated to operational taxonomic units (OTUs) using the USEARCH pipeline (http://www.drive5.com/usearch/)(24). The Mothur algorithm was used to compare representative sequence of each OTU with the non-redundant SILVA database. Alpha and beta diversity analyses were performed using the determined OTUs and UniFrac distances, respectively, and implemented in QIIME.

\section{Statistical analysis}

Each piglet was used as a statistical unit. The significance of differences was analyzed by one-way ANOVA using General Linear Model procedures in SAS (SAS Institute, Cary, NC, USA), followed by Duncan's multiple range tests. Significance was considered at $P<0.05$.

\section{Results}

\section{Rectal temperature and intestinal $\mathrm{pH}$ value}

Piglets challenged with LPS in the $\mathrm{CL}, \mathrm{OL}, \mathrm{AL}$, and OAL groups trended toward higher rectal temperatures than did piglets without LPS challenge in the $C$ group (Fig. 1A). Piglets in the $C L, O L, A L$, and OAL groups had significantly higher stomach $\mathrm{pH}$ values than did piglets in the $\mathrm{C}$ group $(P<0.05)$ (Fig. 1B). Piglets in the AL and OAL groups had significantly lower duodenum, jejunum, and ileum $\mathrm{pH}$ values than did piglets in the $\mathrm{C}$ groups $(P<0.05)$. Inclusion of antibiotics or antibiotics plus olive extract in the diet significantly lowered the $\mathrm{pH}$ value in the jejunum and ileum compared with the $\mathrm{CL}$ groups $(P<0.05)$ (Fig. 1D, E). There was no significant difference in colon $\mathrm{pH}$ values in piglets among different treatment groups $(P>0.05)$ (Fig. 1F).

\section{Serum Biochemical Characteristics}


The LPS challenge significantly lowered serum glucose levels in the CL group compared with the $C$ group $(P<0.05)$. Diet inclusion of olive extract significantly increased the serum glucose in OL piglets when compared with $\mathrm{CL}$ group piglets $(P<0.05)$. AL group piglets did not significantly differ from $\mathrm{CL}$ group piglets $(P>0.05)$. However, OAL group piglets, fed a diet with antibiotics plus olive extract, had significantly higher glucose than did the $\mathrm{CL}$ group piglets $(P<0.05)$ (Fig. $2 \mathrm{~A})$. The serum total protein content was significantly lower in $\mathrm{CL}, \mathrm{OL}$, and $\mathrm{AL}$ group piglets than in $\mathrm{C}$ group piglets $(P<0.05)(\mathrm{Fig}$. $2 \mathrm{~B})$. Serum total protein content was significantly lower in AL group piglets than in OAL group piglets $(P<$ $0.05)$ (Fig. 2B). The piglets in OL groups had significantly lower total serum cholesterol than did piglets in the $\mathrm{CL}$ groups $(P<0.05)$ (Fig. $2 \mathrm{C})$.

\section{Serum Antioxidant Capacity}

Compared with those in the $\mathrm{C}$ group, after i.p. injection of LPS, piglets in the $\mathrm{CL}$ group had significantly lower GSH-Px $(P<0.05)$ and SOD $(P<0.05)$ levels and higher MDA $(P<0.05)$ and NO $(P<0.05)$ levels in the serum (Fig. 3A-D). However, compared with the $\mathrm{CL}$ group, the piglets in $\mathrm{OL}, \mathrm{AL}$, and $\mathrm{OAL}$ groups had significantly higher GSH-Px $(P<0.05)$ and SOD $(P<0.05)$ levels and lower MDA $(P<0.05)$ and NO $(P<$ $0.05)$ levels in the serum (Fig. 3A-D). There was no significant difference in these four parameters in serum of piglets from $\mathrm{OL}, \mathrm{AL}$, and OAL groups $(P>0.05)$ (Fig. 3A-D).

Compared with the $\mathrm{C}$ group, the piglets in the $\mathrm{CL}$ group had significantly higher levels of $\operatorname{LDH}(P<0.05)$, $\operatorname{ALT}(P<0.05)$, and AST $(P<0.05)$ and lower ALB levels $(P<0.05)$ in the serum (Fig. 3E-H). Compared with the $\mathrm{CL}$ group, the piglets in the $\mathrm{OL}, \mathrm{AL}$, and $\mathrm{OAL}$ groups had significantly lower serum $\mathrm{LDH}(P<0.05), \mathrm{ALT}$ $(P<0.05)$, and AST $(P<0.05)$ levels and higher ALB $(P<0.05)$ levels in the serum (Fig. 3E-H). The piglets in the OL and OAL groups were fed diets containing olive extract and had significantly lower serum LDH $(P<0.05)$ levels than did those in the AL groups (Fig. 3E).

\section{Serum Inflammatory Factor And Spleen Index}

LPS administration significantly increased the serum concentration of TNF-a $(P<0.05)$ and IL-6 $(P<0.05)$ in the $\mathrm{CL}$ group compared with the control group (Fig. 4A, B). Compared with those in the $\mathrm{CL}$ group, piglets in the $\mathrm{OL}, \mathrm{AL}$, and OAL groups had significantly lower serum TNF-a $(P<0.05)$ and IL-6 $(P<0.05)$ levels and significantly higher IL-10 $(P<0.05)$ levels (Fig. 4A-C). There were no significant differences in serum TNF-a, IL-6, and IL-10 levels in piglets from OL, AL, and OAL groups $(P>0.05)$ (Fig. 4A-C). Piglets challenged with LPS in the CL group had significantly higher spleen index than did $C$ group piglets $(P<$ 0.05 , Fig. 4D). Spleen index values of piglets in $\mathrm{OL}, \mathrm{AL}$, and $\mathrm{OAL}$ groups did not significantly differ from those in $\mathrm{CL}$ group piglets $(P>0.05$, Fig. 4D).

\section{Intestinal Integrity And Histomorphology}


The DAO and D-xylose was determined as indicators of the intestinal integrity. CL group piglets challenged with LPS had significantly higher serum DAO and D-xylose levels than did group $C$ piglets $(P<$ 0.05 , Fig. $5 A, B)$. The OL and OAL group piglets had significantly lower DAO and D-xylose concentrations than did $C L$ group piglets $(P<0.05$, Fig. $5 \mathrm{~A}, \mathrm{~B})$. The villus height and crypt depth, based on H\&E staining, are shown in Fig. 6. The duodenum and ileum of piglets in control, OL, AL and OAL groups showed regular structures, while LPS stimulation destroyed the intestinal villus structure in the duodenum and ileum of piglets in the $\mathrm{CL}$ group (Fig. 6A, B). The duodenum and ileum of piglets in the $\mathrm{CL}$ group had significantly lower villus height $(P<0.05)$, higher crypt depth $(P<0.05)$ and lower villus height/crypt depth ratios $(P<0.05)$ than those of piglets in the control group (Fig. 6C, D). However, compared with those of the $\mathrm{CL}$ group, the duodenum and ileum of piglets in $\mathrm{OL}, \mathrm{AL}$, and $\mathrm{OAL}$ groups had significantly higher villi $(P<0.05)$, lower crypt depth $(P<0.05)$, and higher villus height/crypt depth ratios $(P<0.05)$ with the exception of ileum villus height in $\mathrm{AL}$ and OAL groups $(P>0.05)$ (Fig. 6C, D).

\section{Piglet Fecal Bacterial Communities}

The relative abundance of fecal microbiota at the family (Fig. 7A) and genus levels (Fig. 7B) were examined in pig fecal matter. There were $777,694,763,680$, and 733 OTUs obtained from the feces of piglets in C, CL, OL, AL, and OAL groups, respectively. Among these OTUs, 448 OTUs were shared and 197 OTUs were isolated (Fig. 7C). We performed LEfSe analysis to identify the significant ranking of abundant bacterial taxa among $\mathrm{C}, \mathrm{CL}, \mathrm{OL}, \mathrm{AL}$, and OAL groups. The cladogram highlighted 19 important bacterial taxa (Fig. 7D), and their significant effects were displayed using LDA scores (Fig. 7E). The threshold for the logarithmic LDA score for biomarkers was set at 2.0. The biomarker in C was p_251_o5 (c). The biomarkers in CL were Eubacterium_coprostanoligenes_group (g) and T34(p). The biomarkers in OL were Megasphaera (j), Veillonellaceae $(\mathrm{k})$, and Selenomonadales (I). The biomarkers in AL were Lactobacillus (d), Lactobacillaceae (e), and Lactobacillales (f), which formed one branch, and Treponema_2 (q), Spirochaetaceae (r), and Spirochaetales (s) formed a second branch. Muribaculaceae (a), Tannerellaceae (b), Ruminococcaceae (h), Anaerovibrio (i), Succinivibrio (m), Succinivibrionaceae (n), and Aeromonadales (o) were biomarkers in OAL (Fig. 7E).

Chao 1 and Shannon and Simpson index values, which reflect species richness and evenness, did not differ among the five treatment groups (Fig. 8A-C). At the family level (Fig. 8D), there was no significant difference in relative abundance of Muribaculaceae, Tannerellaceae, Tannerellaceae, and Ruminococcaceae between the $\mathrm{C}$ and $\mathrm{CL}$ groups, while the $\mathrm{CL}$ group had a significantly higher relative abundance of Veillonellaceae and T34 $(P<0.05)$ and a lower relative abundance of Spirochaetaceae $(P<$ $0.05)$ than did the $C$ group. The relative abundance of Muribaculaceae was significantly lower in $C L$ group piglets than in OAL group piglets $(P<0.05)$. The relative abundance of Tannerellaceae was significantly lower in $\mathrm{CL}$ group piglets than in $\mathrm{OL}, \mathrm{AL}$, and $\mathrm{OAL}$ group piglets $(P<0.05)$. The relative abundance of Lactobacillaceae was significantly lower in $\mathrm{CL}$ group piglets than in $\mathrm{OL}$ and $\mathrm{AL}$ group piglets $(P<0.05)$. The relative abundance of Ruminococcaceae was significantly lower in $\mathrm{OL}$ group piglets than in $\mathrm{CL}$ group piglets $(P<0.05)$. There was no significant difference in the relative abundance 
of Veillonellaceae in the intestines among the $\mathrm{CL}, \mathrm{OL}, \mathrm{AL}$, and $\mathrm{OAL}$ group piglets $(P>0.05)$. The relative abundance of $T 34$ was significantly lower in the $\mathrm{OL}, \mathrm{AL}$, and $\mathrm{OAL}$ group piglets than in the $\mathrm{CL}$ group piglets $(P<0.05)$. The relative abundance of Spirochaetaceae was significantly lower in $\mathrm{CL}$ group piglets than in $\mathrm{OL}$ and $\mathrm{AL}$ group piglets $(P<0.05)$.

At the genus level (Fig. 8E), LPS challenge induced a significantly higher relative abundance of Alloprevotella $(P<0.05)$ and a lower abundance of Clostridium_sensu_stricto_1, Lachnospiraceae_NK4A136_group, and Succinivibrio $(P<0.05)$ in CL group piglets. The relative abundance of Alloprevotella was significantly lower in $\mathrm{OL}, \mathrm{AL}$ and $\mathrm{OAL}$ group piglets than in $\mathrm{CL}$ group piglets $(P<0.05)$. Dietary inclusion of olive extract in the OL group had significantly higher relative abundance of Clostridium_sensu_stricto_ 1 than did the $\mathrm{CL}$ group $(P<0.05)$. The relative abundance of Lactobacillus was significantly lower in $\mathrm{CL}$ group piglets than in $\mathrm{OL}$ and $\mathrm{AL}$ group piglets $(P<0.05)$. There was no significant difference in the relative abundance of Lachnospiraceae_NK4A136_group in the intestine of $\mathrm{CL}, \mathrm{OL}, \mathrm{AL}$, and $\mathrm{OAL}$ group piglets $(P>0.05)$. The $\mathrm{OL}$ and $\mathrm{OAL}$ group piglets had significantly higher relative Succinivibrio abundance than did the CL group piglets $(P<0.05)$. There was no significant difference in relative Sutterella abundance between $\mathrm{C}$ and $\mathrm{CL}$ group piglets $(P>0.05)$. The relative abundance of Sutterella was significantly lower in CL group piglets than in the OL and OAL groups $(P<$ 0.05). The relative Terrisporobacter abundance was significantly lower in the $\mathrm{CL}$ group piglets than in $\mathrm{OL}$ group piglets $(P<0.05)$. The OL and AL group piglets had significantly higher relative Treponema_2 abundance than did the $\mathrm{CL}$ groups $(P<0.05)$.

\section{Discussion}

In this study, weaned piglet that underwent LPS challenge were used as an experimental model of inflammation. The body temperature of healthy piglets is between $38.6^{\circ} \mathrm{C}$ and $39.7^{\circ} \mathrm{C}(25)$, and inflammation increases the body temperature. Our test results show that the body temperature trended upwards after LPS treatment $(P=0.0965)$, and that all temperatures were higher than $39.7^{\circ} \mathrm{C}$ after LPS treatment. This result indicates that LPS had activated the immune response and inflammation to increase body temperature. Lower gastrointestinal $\mathrm{pH}$ is beneficial for the persistence and colonization of beneficial intestinal bacteria. Our results show that the $\mathrm{pH}$ of gastric contents in four groups induced by LPS was significantly increased compared with that in the control group, indicating that LPS may induce secretion of acid in the stomach. Compared with the $\mathrm{CL}$ group, the dietary inclusion of olive extract, antibiotics, or olive extract and antibiotics reduced the intestinal tract $\mathrm{pH}$ to a certain extent, which also may lay the foundation for the growth environment of beneficial bacteria.

Blood glucose is the main energy source for body activities. LPS challenge induced an inflammatory response and oxidant stress, which may lead to the consumption of glucose as energy and cause significantly lower blood glucose in the CL group. Blood glucose levels in OL and OAL groups were restored to levels similar to those observed in the control without LPS challenge. This may contribute to the anti-inflammatory and anti-oxidant effects of the olive extract, which may allow for resist of LPS stimulation to maintain stable blood glucose concentration. Serum total protein is significantly reduced in 
piglets after stimulation with LPS, which may be attributed to the acute inflammation induced by LPS in $\mathrm{CL}, \mathrm{OL}$, and $\mathrm{AL}$ groups. The OAL group did not significantly differ from the $\mathrm{C}$ group, indicating that in the olive and antibiotic group inflammation induced by LPS could be alleviated and influence the level of total protein in serum. Ampelopsin, a plant extract from ampelopsis grossedentata, has antioxidant activity, alleviates the inflammatory response induced by LPS, and maintains glucose and ALB levels in serum(26).

Olive oil was shown to significantly reduce the cholesterol content in serum of rabbits fed high cholesterol diet in an antithrombotic study(27). An in vitro and in vivo study of lipid metabolism regulation showed that Chinese olive extract effectively reduces the level of serum cholesterol (28). Olive extract significantly decreased total cholesterol in plasma from 2.1 to $1.3 \mathrm{mmol} / \mathrm{L}$ in mice fed highcarbohydrate and high-fat diets(15). Consistently, in our study, the diet containing olive extract, in the OL and OAL groups, had lower total serum cholesterol levels than in the $\mathrm{C}$ and $\mathrm{CL}$ groups. Taken together, these results indicate that olive extract has the capacity to reduce serum cholesterol.

Olive leaf and its constituents have been previously described to have antioxidant and free radical scavenging properties(29). Weaning of mammals involves many complicated events, including the influence of environmental and dietary pressures on intestinal development and adaptability, which can lead to inflammation(30). MDA is a reliable indicator of oxidative stress, and can reflect the degree of damage caused by reactive oxygen metabolites in cells(31). SOD, GSH-Px, and NO play key roles in the defense against reactive oxygen species activated by oxidative stress injury(32). Our results show that $\mathrm{OL}, \mathrm{AL}$, and OAL significantly increase the GSH-Px and SOD levels and reduce the MDA and NO levels in serum after LPS induction. Previous results also showed that olive extract significantly decreases plasma MDA, a marker of lipid peroxidation, from 32.2 to $23.6 \mu \mathrm{mol} / \mathrm{L}$ in mice fed a high-carbohydrate and highfat diet(15). These results are consistent with previous results which showed that the levels of serum MDA and NO are increased, and SOD and GSH-Px levels are decreased after LPS injection, while the carnosic acid treatment alleviated the influence of LPS(33). Therefore, olive extract can exert certain role in alleviating the oxidant stress induced by LPS.

ALT and AST are important indicators of liver function(34). LDH is an important enzyme in sugar metabolism and is a sensitive indicator of cell membrane damage(35). The significantly higher serum levels of ALT, AST, and LDH observed in CL group piglets indicate that LPS challenge damages the liver. However, the dietary inclusion of olive extract, antibiotics, or olive extract plus antibiotics alleviates LPSinduced liver damage as observed by significantly lower levels of ALT, AST, and LDH in serum from OL, $A L$, and OAL group piglets. Consistent with our result, it was shown that LPS significantly increases ALT and AST levels in serum, while hesperidin treatment reduces the elevated serum AST and ALT(36). Limited data are available on the effects of olive leaf, or its major constituents, on the liver. Olive extract significantly decreases plasma ALT, AST, and LDH levels in mice fed a high-carbohydrate and high-fat diet, indicating the hepatoprotective effects of OLE(15). Due to liver damage, ALB content in serum is reduced, while $\mathrm{OL}, A L$, and OAL weaken the degree of ALB reduction in serum(37), indicating that olive extract can relieve liver damage caused by LPS to a certain extent. 
IL-10 has immunosuppressive and anti-inflammatory effects, which are opposite to the effects of IL-6 and TNF-a(38). Our results indicate that olive extract alleviates the inflammatory reaction induced by LPS. Consistently, a previous study found that hypericum triquetrifolium extract significantly inhibited TNF- $\alpha$ and IL-6 expression and secretion and significantly elevated IL-10 secretion(39). Olive leaf-derived polyphenols exert an improtant role in preventing inflammation and oxidative damage-induced primary myocardial insult(15). Similarly, the olive leaf and olive oil treatments decreased oxidative stress and inflammation, TNF-a levels, and increased IL-10 levels(40). These results show that olive extract has antiinflammatory properties.

Villus height, crypt depth, and VCR (villus height to crypt depth ratio) are criteria that reflect the general morphology of the intestinal tract(20,41). LPS treatment led to a rise in the villus end epithelium, reduced the villus height in the duodenum, jejunum, and ileum mucosa, and increased the depth of the ileum mucosa crypt $(20,42)$. Our results show that LPS stimulation decreased the villus height of the duodenum, jejunum, and ileum, increased the crypt depth and reduced the VCR. However, supplementation with olive extract (OL) alleviated LPS-induced inflammation, thus restoring villus height and reducing crypt depth.

The observed increase in DAO and D-xylose levels in piglets challenged with LPS alone suggests that increased intestinal permeability was achieved using this experimental model(43). This abnormal "leaky" gut was associated with destroyed villus structure. Dietary olive extract attenuated the LPS-induced rise in DAO and D-xylose levels in the sera of piglets in the OL and OAL groups, suggesting that olive extract may restore intestinal integrity, preventing DAO and D-xylose from crossing the intestinal barrier and entering the circulation. Previously, LPS injection increased the crypt depth in the ileum and jejunum, and reduced the VCR. However, treatment with procyanidin alleviated LPS-mediated destruction of small intestine morphology(44). Dietary supplementation with resveratrol significantly increased villus height and villus height/crypt depth in piglets(45). Morphological damage induced by LPS stimulation in the small intestine includes hemorrhage caused by ulcer and erosion, lumen stenosis, tube wall perforation, and long villi tip damage and plaques(46). In our study, the OL group showed less breakage in the morphology of the small intestine, indicating that, to a certain extent, olive extract could alleviate the damage induced by LPS to ensure the integrity of intestinal morphology and structure.

Venn diagram analysis revealed that LPS treatment reduced the relative OUTs in piglets, while, in the OL group, olive extract supplementation maintains OUTs numbers similar to those of the control group. Compared with the $\mathrm{CL}$ group, olive extract restored the diversity of intestinal microbiota in OL group weaned piglets. Clostridium and Treponema are key participants in nutrition metabolism including in carbohydrate fermentation and polysaccharide and steroid metabolism, and are the key to maintaining the normal physiological function of the intestinal tract $(47,48)$. Several members of the Clostridium family are involved in carbohydrate degradation and metabolic efficiency improvement, which are enriched in feces samples from pigs with higher feed utilization capacity $(49,50)$. Our results indicate that olive extract could increase the relative abundance of Sutterella and Clostridium_sensu_stricto_ 1 in OL group piglets. Clostridium bacteria involved in butyric acid production and mucin degradation are related 
to improved gastrointestinal tract health in pigs(51). Therefore, the relative abundance of Clostridium_sensu_stricto_ 1 in the OL group may enhance epithelial barrier function(52).

Lactobacillus is considered a carbohydrate-utilizing bacterium, and has many genes encoding various functional capabilities related to carbohydrate transportation and utilization(53). Our results suggest that there is a significantly higher abundance of Lactobacillus in piglets treated with olive extract than in $\mathrm{CL}$ group piglets. The reduced small intestine $\mathrm{pH}$ in the group with olive extract supplementation may aid in increasing the abundance of Clostridium and Lactobacillus in the intestinal tract and exert a positive effect on pig intestinal health. These results indicate that the addition of olive extract could increase beneficial bacteria in the intestinal tract and may alleviate the deleterious influence induced by LPS treatment.

\section{Conclusions}

The dietary inclusion of olive extract improved anti-oxidative and anti-inflammatory abilities in weaned piglets. Dietary inclusion of olive extract protected the intestinal morphology from LPS-induced damage by increasing the length of intestinal villi and reducing the crypt depth. Dietary inclusion of olive extract decreased the intestinal $\mathrm{pH}$ in the intestine and increased the relative abundance of beneficial bacteria including Lactobacillus and Clostridium. Therefore, olive extract can be used as a dietary ingredient to improve the anti-oxidant and anti-inflammatory activity to benefit health of piglet.

\section{Abbreviations}

ALB, albumin; ALT, alanine aminotransferase; AST, aspartate aminotransferase; DAO, diamine oxidase; GSH-Px, glutathione peroxidase; IL, interleukin; LDA, linear discriminant analysis; LDH, lactate dehydrogenase; LPS, lipopolysaccharide; MDA, malondialdehyde; NO, nitric oxide; OTUs, operational taxonomic units; SOD, superoxide dismutase; T-CHO, total cholesterol; TNF-a, tumor necrosis factor-a.

\section{Declarations}

\section{Acknowledgements}

The authors thank Mr. Feng Ye for assistance with piglet feeding.

\section{Author's contributions}

$\mathrm{HFW}, \mathrm{YZ}$ and MLH designed the study, analyzed the data, and wrote the manuscript; $Y Z$ and ZXD performed the experiments; JXL and XML provided expert advice on the experimental design, interpretation of data, and editing of the manuscript. All authors read and approved the final manuscript.

\section{Funding}


This study was financially supported by the National Natural Science Foundation of China (31672430), the Natural Science Foundation of Zhejiang Province (Z19C170001) and Lucta innovation fund.

\section{Availability of data and materials}

The data were shown in the main manuscript.

\section{Ethics approval and consent to participate}

This trial was registered at the Zhejiang University (http://www.lac.zju.edu.cn) as No. ZJU20170529.

\section{Competing interests}

The authors declare that the research was conducted in the absence of any commercial or financial relationships that could be construed as a potential conflict of interest.

\section{Author details}

${ }^{1}$ College of Animal Science, Zhejiang University, The Key Laboratory of Molecular Animal Nutrition, Ministry of Education, Hangzhou 310058, China. ${ }^{2}$ Innovation Division, Lucta (Guangzhou) Flavours Co. Ltd., Guangzhou, 510530, China. ${ }^{3}$ Department of Biomedical Sciences and Pathobiology, Virginia Tech, Blacksburg, VA 24061, USA

\section{References}

1. Lee IK, Kye YC, Kim G, Kim HW, Gu MJ, Umboh J, et al. Stress, nutrition, and intestinal immune responses in pigs-a review. Asian Australasian Journal of Animal Science. 2016;29(8):1075-82.

2. Buonocore G, Perrone S, Tataranno ML. Oxidative stress in the newborn. Oxidative Medicine and Cellular Longevity. 2017:1094247.

3. Hu CH, Xiao K, Luan ZS, Song J. Early weaning increases intestinal permeability, alters expression of cytokine and tight junction proteins, and activates mitogen-activated protein kinases in pigs 1 . J Anim Sci. 2013;91(3):1094-101.

4. Yin J, Wu MM, Xiao H, Ren WK, Duan JL, Yang G, et al. Development of an antioxidant system after early weaning in piglets. J Anim Sci. 2014;92:612-9.

5. Duan JL, Yin J, Ren WK, Liu T, Cui ZJ, Huang XG, et al. Dietary supplementation with I-glutamate and I-aspartate alleviates oxidative stress in weaned piglets challenged with hydrogen peroxide. Amino Acids. 2016;48:53-64.

6. Liu Y, Chen F, Odle J, Lin X, Jacobi SK, Zhu H, et al. Fish oil enhances intestinal integrity and inhibits TLR4 and NOD2 signaling pathways in weaned pigs after LPS challenge. J Nutr. 2012;142(11):2017-24. PubMed PMID: 23014495.

7. Fasano A, Sheadonohue T. Mechanisms of disease: the role of intestinal barrier function in the pathogenesis of gastrointestinal autoimmune diseases. Nature Clinical Practice Gastroenterology 
hepatology. 2005;2(9):416-22.

8. Yin J, Ren WK, Liu G, Duan JL, Yang G, Wu L, et al. Birth oxidative stress and the development of an antioxidant system in newborn piglets. Free Radical Res. 2013;47(12):1027-35.

9. Zhu LH, Xu JX, Zhu SW, Cai X, Yang SF, Chen XL, et al. Gene expression profiling analysis reveals weaning-induced cell cycle arrest and apoptosis in the small intestine of pigs. J Anim Sci. 2014;92:996-1006.

10. Ghanbari R, Anwar F, Alkharfy KM, Gilani AH, Saari N. Valuable nutrients and functional bioactives in different parts of Olive (Olea europaea L.)-A Review. Int J Mol Sci. 2012;13:3291-340.

11. Boskou D. Olive Fruit, Table Olives, and Olive Oil Bioactive Constituents. In: Boskou D, editor. Olive and Olive Oil Bioactive Constituents: AOCS Press; 2015. p. 1-30.

12. Beauchamp GK, Keast RS, Morel D, Lin J, Pika J, Han Q, et al. Phytochemistry: ibuprofen-like activity in extra-virgin olive oil. Nature. 2005;437(7055):45-6.

13. Knoops K, Groot L, Kromhout D, Perrin A-E, Moreiras-Varela O, Menotti A, et al. Mediterranean diet, lifestyle factors, and 10-year mortality in elderly european men and women. JAMA: Journal of the American Medical Association. 2004;10(01):292:1433-9.

14. Erbay Z, Icier F. The importance and potential uses of olive leaves. Food Reviews International. 2010;26(4):319-34.

15. Poudyal H, Campbell F, Brown L. Olive leaf extract attenuates cardiac, hepatic, and metabolic changes in high carbohydrate-, high fat-fed rats. J Nutr. 2010;140:946-53.

16. Lesage-Meessen L, Navarro D, Maunier S, Sigoillot JC, Lorquin J, Delattre M. Simple phenolic content in olive oil residues as a function of extraction systems. Food Chem. 2001;75:501-7.

17. Amro B, Aburjai T, Al-Khalil S. Antioxidative and radical scavenging effects of olive cake extract. Fitoterapia. 2002;73:456-61.

18. Johnson RW, von Borell E. Lipopolysaccharide-induced sickness behavior in pigs is inhibited by pretreatment with indomethacin. Journal of animal science. 1994 1994/02//;72(2):309-14. PubMed PMID: 8157515. eng.

19. Alscher KT, Phang PT, McDonald TE, Walley KR. Enteral feeding decreases gut apoptosis, permeability, and lung inflammation during murine endotoxemia. Am J Physiol Gastrointest Liver Physiol. 2001 2001/08//;281(2):G569-76. PubMed PMID: 11447038. eng.

20. Liu Y, Huang J, Hou Y, Zhu H, Zhao S, Ding B, et al. Dietary arginine supplementation alleviates intestinal mucosal disruption induced by Escherichia coli lipopolysaccharide in weaned pigs. $\mathrm{Br} \mathrm{J}$ Nutr. 2008;100(3):552-60.

21. Lin CJ, Chiu CC, Chen YC, Chen ML, Hsu TC, Tzang BS. Taurine attenuates hepatic inflammation in chronic alcohol-fed rats through inhibition of TLR4/MyD88 signaling. J Med Food. 2015;18(12):1291.

22. Munro BH. Manual of histologic staining methods of the armed forces institute of pathology. Pathology. 1971 1971/01/01/;3(3):249. 
23. Caporaso JG, Kuczynski J, Stombaugh J, Bittinger K, Bushman FD, Costello EK, et al. QIIME allows analysis of high-throughput community sequencing data. Nat Methods. 2010;7(5):335-6. 2010/05/01.

24. Edgar RC. UPARSE: highly accurate OTU sequences from microbial amplicon reads. Nat Methods. 2013;2013(10):996-8. /10/01;10(.

25. Murray Pullar E. The rectal temperature in normal and infected pigs. British Veterinary Journal. 1949 1949/12/01/;105(12):437-53.

26. Hou X, Wang T, Ahmad H, Xu Z. Ameliorative effect of ampelopsin on LPS-induced acute phase response in piglets. Journal of Functional Foods. 2017;35:489-98.

27. De La Cruz JP, Villalobos MaA, Carmona JA, Martín-Romero M, Smith-Agreda JMa, de la Cuesta FS. Antithrombotic potential of olive oil administration in rabbits with elevated cholesterol. Thromb Res. 2000;100(4):305-15.

28. Yeh YT, Cho YY, Hsieh SC, Chiang AN. Chinese olive extract ameliorates hepatic lipid accumulation in vitro and in vivo by regulating lipid metabolism. Scientific reports. 2018;8(1):1057. PubMed PMID: 29348600. Pubmed Central PMCID: 5773498.

29. Lee OH, Lee BY, Lee J, Lee HB, Son JY, Park CS, et al. Assessment of phenolics-enriched extract and fractions of olive leaves and their antioxidant activities. Biores Technol. 2009;100:6107-13.

30. Lallès JP, Bosi P, Smidt H, Stokes CR. Weaning - A challenge to gut physiologists. Livestock Science. 2007;108(1):82-93.

31. Ahmed MAE. Amelioration of nandrolone decanoate-induced testicular and sperm toxicity in rats by taurine: Effects on steroidogenesis, redox and inflammatory cascades, and intrinsic apoptotic pathway. Toxicology and Applied Pharmacology. 2015 2015/02/01/;282(3):285-96.

32. Guzik TJ, Korbut R, Adamek-Guzik T. Nitric oxide and superoxide in inflammation and immune regulation. Journal of Physiology Pharmacology. 2004;54(4):469-87.

33. Xiang Q, Liu Z, Wang Y, Xiao H, Wu W, Xiao C, et al. Carnosic acid attenuates lipopolysaccharideinduced liver injury in rats via fortifying cellular antioxidant defense system. Food Chemical Toxicology. 2013;53:1-9.

34. Hammes TO, Leke R, Escobar TDC, Fracasso LB, Meyer FS, Andrades M, et al. Lactobacillus rhamnosus $\mathrm{GG}$ reduces hepatic fibrosis in a model of chronic liver disease in rats. Nutricion hospitalaria. 2017 Jun 5;34(3):702-9. PubMed PMID: 28627210. Epub 2017/06/20. eng.

35. Huang X, Liu Y, Song FR, Liu ZQ, Liu SY. Studies on principal components and antioxidant activity of different Radix Astragali samples using high-performance liquid chromatography/electrospray ionization multiple-stage tandem mass spectrometry. Talanta. 2009;78(3):1090-101.

36. Kaur G, Tirkey N, Chopra K. Beneficial effect of hesperidin on lipopolysaccharide-induced hepatotoxicity. Toxicology. 2006 2006/09/21/;226(2):152-60.

37. Huo HZ, Wang B, Liang YK, Bao YY, Gu Y. Hepatoprotective and antioxidant effects of licorice extract against CCl4-induced oxidative damage in rats. Int J Mol Sci. 2011;12(12):6529-43. 
38. Pestka S, Krause CD, Walter MR, Shi Y, Fisher PB. Interleukin-10 and related cytokines and receptors. Annu Rev Immunol. 2004;22(22):929-79.

39. Saad B, Hashem Wa, Farich B, Mahajna S, Azab M, Daragmeh J, et al. Hypericum triquetrifolium extracts modulate IL-6, IL-10 and TNF-a protein and mRNA expression in LPS-activated human peripheral blood mononuclear cells and THP-1-derived macrophages. Medicinal \& Aromatic Plants. 2016 05/01;:S3:004.

40. Ramos MFdP, Oliveira OB, de Barros AdCMM, Razvickas CV, Pessoa EdA, da Silva RF, et al. Comparison of olive leaf, olive oil, palm oil, and omega-3 oil in acute kidney injury induced by sepsis in rats. PeerJ. 2019 2019/07/09;7:e7219.

41. Hampson DJ. Alteration in piglet small intestinal structure at weaning. Res Vet Sci. 1986;40(1):3240.

42. Lobo SM, De Backer D, Sun Q, Tu Z, Dimopoulos G, Preiser J-C, et al. Gut mucosal damage during endotoxic shock is due to mechanisms other than gut ischemia. J Appl Physiol. 2003;95(5):204754.

43. Mao JD, Qi SR, Cui YJ, Dou XX, Luo XM, Liu JX, et al. Lactobacillus rhamnosus GG attenuates lipopolysaccharide-induced inflammation and barrier dysfunction by regulating MAPK/NF-KB signaling and modulating metabolome in the piglet intestine. J Nutr. 2020;150:1313-23.

44. Wu QJ, Wang YQ, Qi YX. The protective effect of procyanidin against LPS-induced acute gut injury by the regulations of oxidative state. SpringerPlus. 2016 2016/09/22;5(1):1645.

45. Chen X, Zeng Z, Huang Z, Chen D, He J, Chen H, et al. Effects of dietary resveratrol supplementation on immunity, antioxidative capacity and intestinal barrier function in weaning piglets. Animal Biotechnology. 2019.

46. Rocha O, Ansari K, Doohan FM. Effects of trichothecene mycotoxins on eukaryotic cells: A review. Food Additives \& Contaminants. 2005 2005/04/01;22(4):369-78.

47. Louis P, Flint HJ. Diversity, metabolism and microbial ecology of butyrate-producing bacteria from the human large intestine. FEMS Microbiol Lett. 2009;294(1):1-8.

48. Flint HJ, Bayer EA, Rincon MT, Lamed R, White BA. Polysaccharide utilization by gut bacteria: potential for new insights from genomic analysis. Nat Rev Microbiol. 2008;6(2):121-31.

49. Mao S, Zhang M, Liu J, Zhu W. Characterising the bacterial microbiota across the gastrointestinal tracts of dairy cattle: membership and potential function. Scientific reports. 2015;5(1):16116.

50. Yang $H$, Huang $X$, Fang $S$, Xin W, Huang L, Chen C. Uncovering the composition of microbial community structure and metagenomics among three gut locations in pigs with distinct fatness. Scientific reports. 2016 2016/06/03;6(1):27427.

51. Levine UY, Looft T, Allen HK, Stanton TB. Butyrate-producing bacteria, including mucin degraders, from the swine intestinal tract. Applied Environmental Microbiology. 2013;79(12):3879-81.

52. Gensollen T, Blumberg, Richard S, lyer, et al. How colonization by microbiota in early life shapes the immune system. Science. 2016. 
53. Cai H, Thompson R, Budinich MF, Broadbent JR, Steele JL. Genome sequence and comparative genome analysis of Lactobacillus casei: Insights into their niche-associated evolution. Genome Biology Evolution. 2010;1:239-57.

\section{Figures}
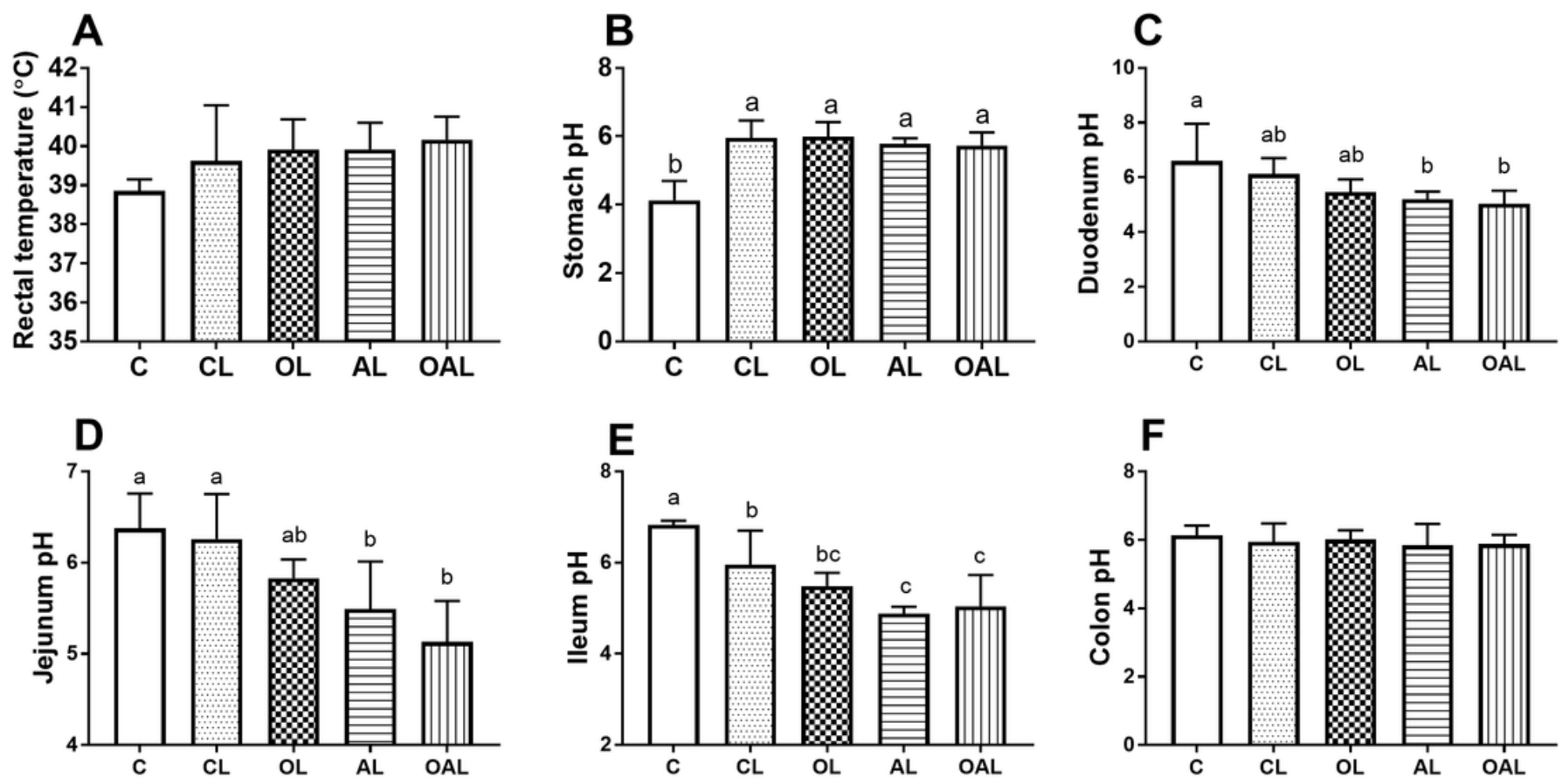

\section{Figure 1}

Rectal temperature and gastrointestinal $\mathrm{pH}$ value in control piglets and those fed basal, antibiotics, olive extract, or antibiotics and olive extract and intraperitoneally (i.p.) injected with LPS. A-F: rectal temperature, stomach, duodenum, jejunum, ileum, and colon $\mathrm{pH}$ value, respectively. Values are shown as means $\pm S D, n=6$. Labeled means with different letters significantly differ, $P<0.05$. $C$, control piglets fed a basal diet; CL, AL, OL, and OAL piglets challenged with LPS (i.p. injection at $20 \mu \mathrm{g} / \mathrm{kg}$ body weight) after being fed with the basal diet, or the basal diet supplemented with antibiotics, olive extract, or antibiotics and olive extract, respectively, for 2 weeks. 

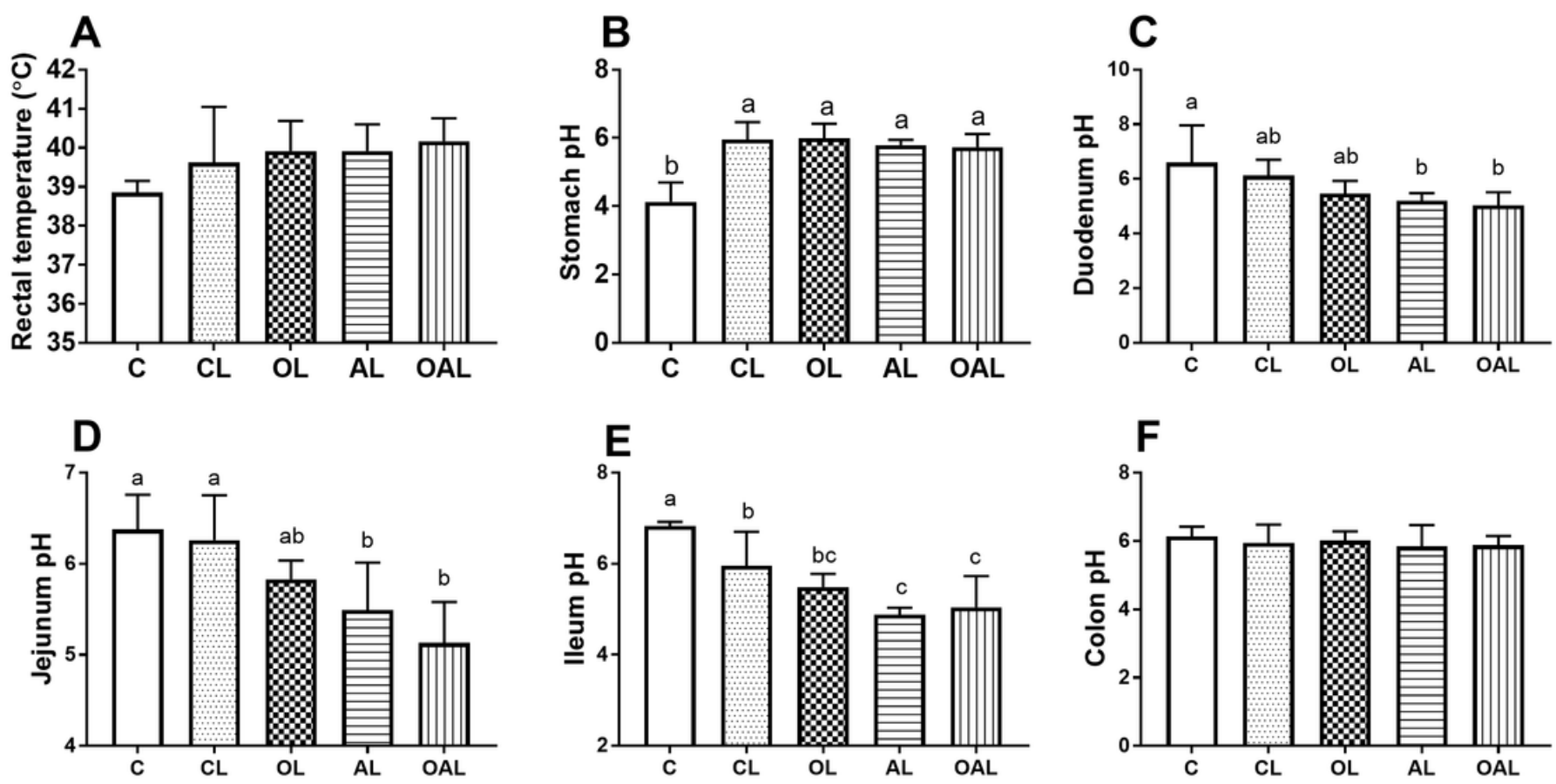

Figure 1

Rectal temperature and gastrointestinal $\mathrm{pH}$ value in control piglets and those fed basal, antibiotics, olive extract, or antibiotics and olive extract and intraperitoneally (i.p.) injected with LPS. A-F: rectal temperature, stomach, duodenum, jejunum, ileum, and colon $\mathrm{pH}$ value, respectively. Values are shown as means $\pm S D, n=6$. Labeled means with different letters significantly differ, $P<0.05$. C, control piglets fed a basal diet; CL, AL, OL, and OAL piglets challenged with LPS (i.p. injection at $20 \mu \mathrm{g} / \mathrm{kg}$ body weight) after being fed with the basal diet, or the basal diet supplemented with antibiotics, olive extract, or antibiotics and olive extract, respectively, for 2 weeks. 

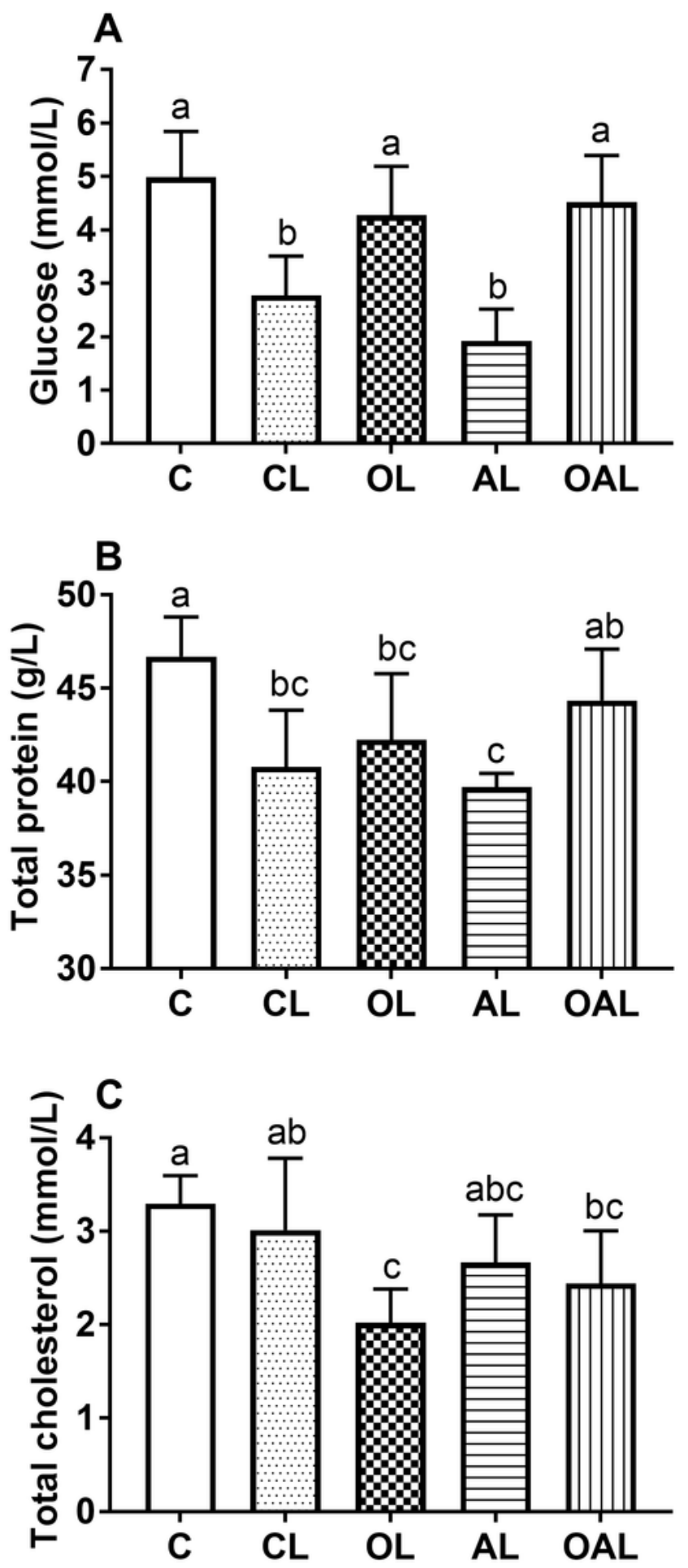

Figure 2

Serum glucose (A), total protein (B), and cholesterol (C) levels in control piglets and those fed basal, antibiotics, olive extract, or antibiotics and olive extract diet and intraperitoneally (i.p.) injected with LPS. Values are shown as means $\pm S D, n=6$. Labeled means with different letters significantly differ, $P<0.05$. $\mathrm{C}$, control piglets fed with a basal diet; $\mathrm{CL}, \mathrm{AL}, \mathrm{OL}$, and $\mathrm{OAL}$, piglets challenged with LPS (i.p. injection at 
$20 \mu \mathrm{g} / \mathrm{kg}$ body weight) after being fed with the basal diet, or the basal diet supplemented with antibiotics, olive extract, or antibiotics and olive extract, respectively, for 2 weeks.
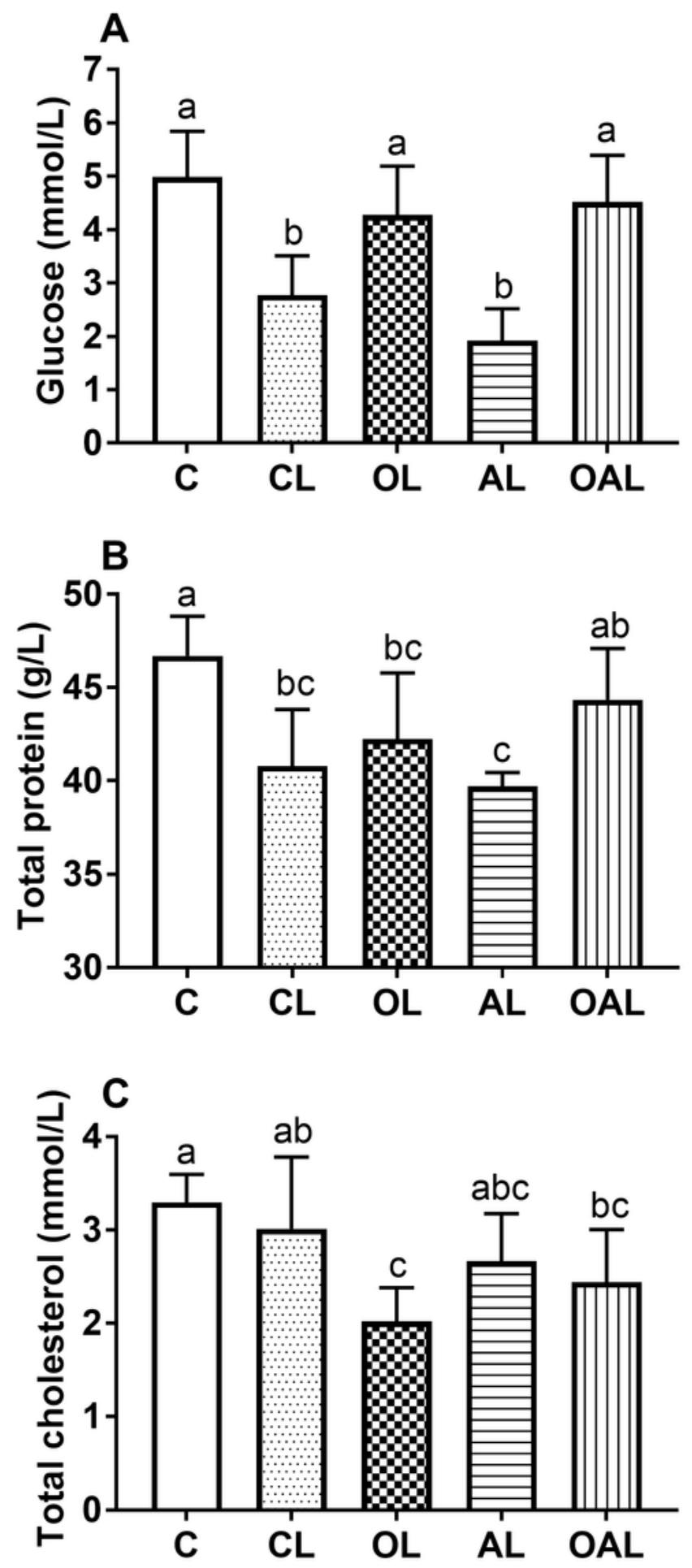

Figure 2

Serum glucose (A), total protein (B), and cholesterol (C) levels in control piglets and those fed basal, antibiotics, olive extract, or antibiotics and olive extract diet and intraperitoneally (i.p.) injected with LPS. Values are shown as means $\pm S D, n=6$. Labeled means with different letters significantly differ, $P<0.05$. 
C, control piglets fed with a basal diet; $\mathrm{CL}, \mathrm{AL}, \mathrm{OL}$, and $\mathrm{OAL}$, piglets challenged with LPS (i.p. injection at $20 \mu \mathrm{g} / \mathrm{kg}$ body weight) after being fed with the basal diet, or the basal diet supplemented with antibiotics, olive extract, or antibiotics and olive extract, respectively, for 2 weeks.
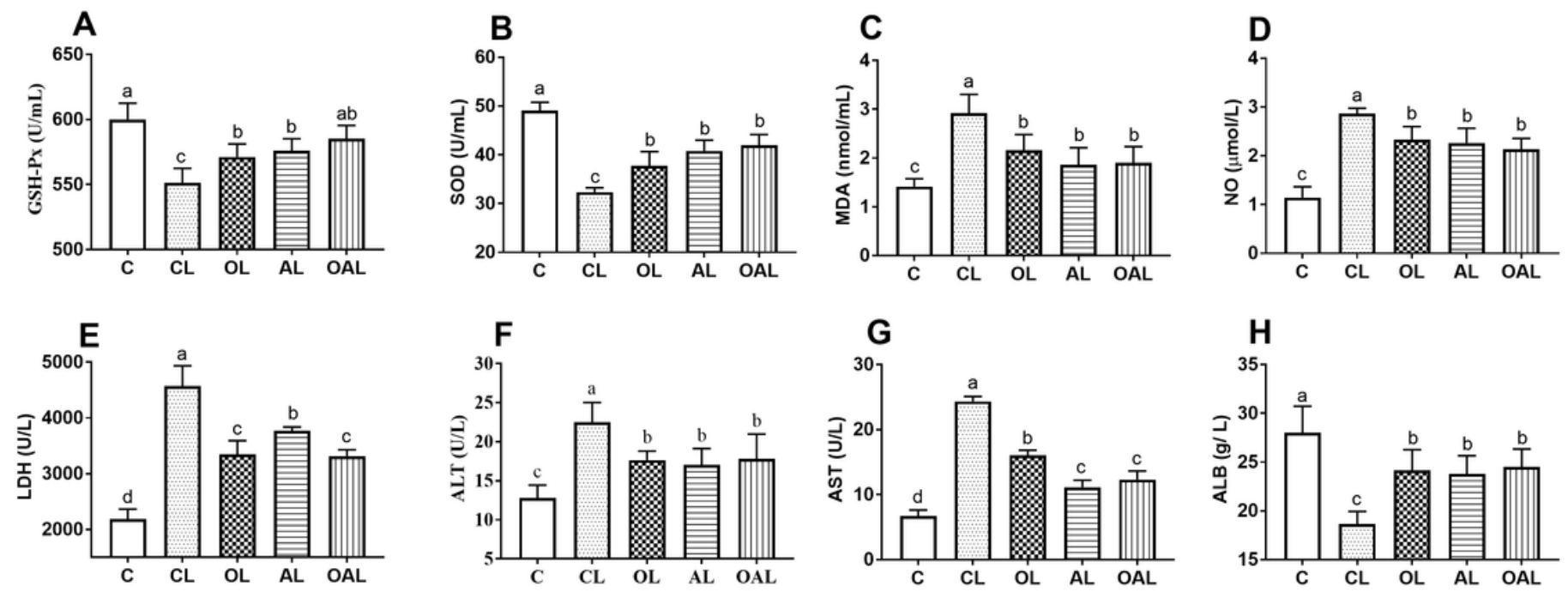

\section{Figure 3}

Serum antioxidant parameters and biochemical enzymes in control piglets and those fed basal, antibiotics, olive extract, or antibiotics and olive extract diet and intraperitoneally (i.p.) injected with LPS. A-H: GSH-Px, SOD, MDA, NO, LDH, ALT, AST, ALB, respectively. Values are shown as means $\pm S D, n=6$. Labeled means with different letters significantly differ, $\mathrm{P}<0.05$. C, control piglets fed with a basal diet; $\mathrm{CL}, \mathrm{AL}, \mathrm{OL}$, and OAL, piglets challenged with LPS (i.p. injection at $20 \mu \mathrm{g} / \mathrm{kg}$ body weight) after being fed with the basal diet, or the basal diet supplemented with antibiotics, olive extract, or antibiotics and olive extract, respectively, for 2 weeks. ALT, alanine aminotransferase; ALB, albumin; AST, aspartate aminotransferase; GSH-Px, glutathione peroxidase; $\mathrm{LDH}$, lactate dehydrogenase; MDA, malondialdehyde; NO, nitric oxide; SOD, superoxide dismutase.
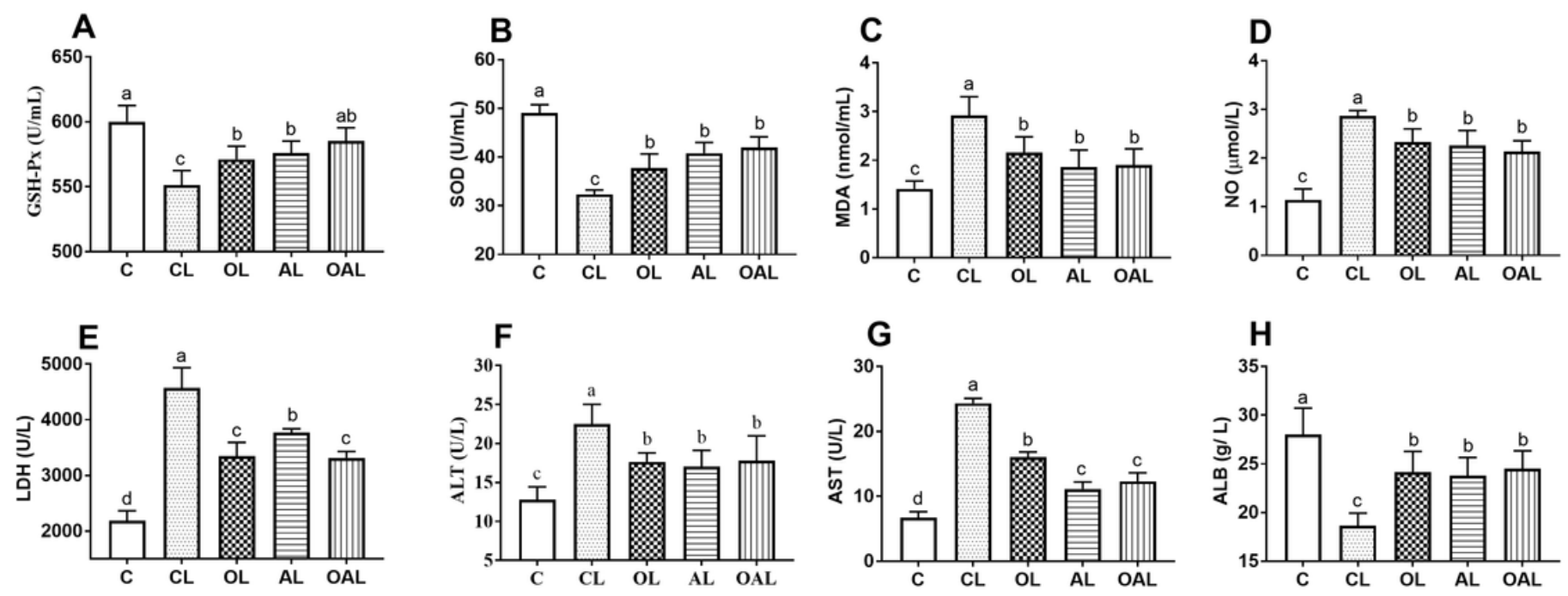
Figure 3

Serum antioxidant parameters and biochemical enzymes in control piglets and those fed basal, antibiotics, olive extract, or antibiotics and olive extract diet and intraperitoneally (i.p.) injected with LPS. A-H: GSH-Px, SOD, MDA, NO, LDH, ALT, AST, ALB, respectively. Values are shown as means $\pm S D, n=6$. Labeled means with different letters significantly differ, $\mathrm{P}<0.05$. C, control piglets fed with a basal diet; $\mathrm{CL}, \mathrm{AL}, \mathrm{OL}$, and OAL, piglets challenged with LPS (i.p. injection at $20 \mu \mathrm{g} / \mathrm{kg}$ body weight) after being fed with the basal diet, or the basal diet supplemented with antibiotics, olive extract, or antibiotics and olive extract, respectively, for 2 weeks. ALT, alanine aminotransferase; ALB, albumin; AST, aspartate aminotransferase; GSH-Px, glutathione peroxidase; LDH, lactate dehydrogenase; MDA, malondialdehyde; NO, nitric oxide; SOD, superoxide dismutase.

A

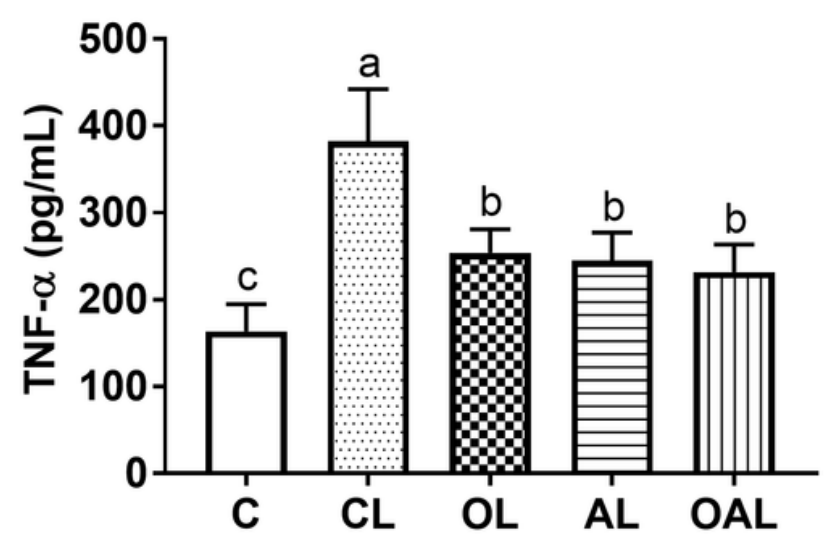

C

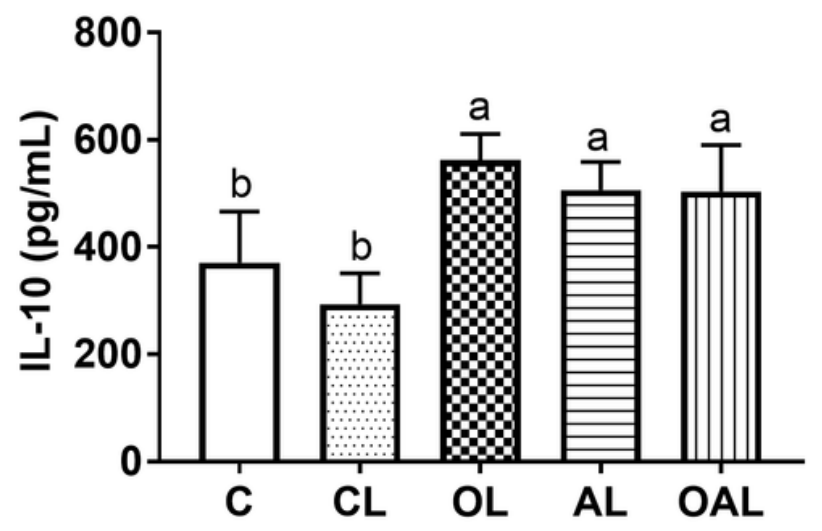

B
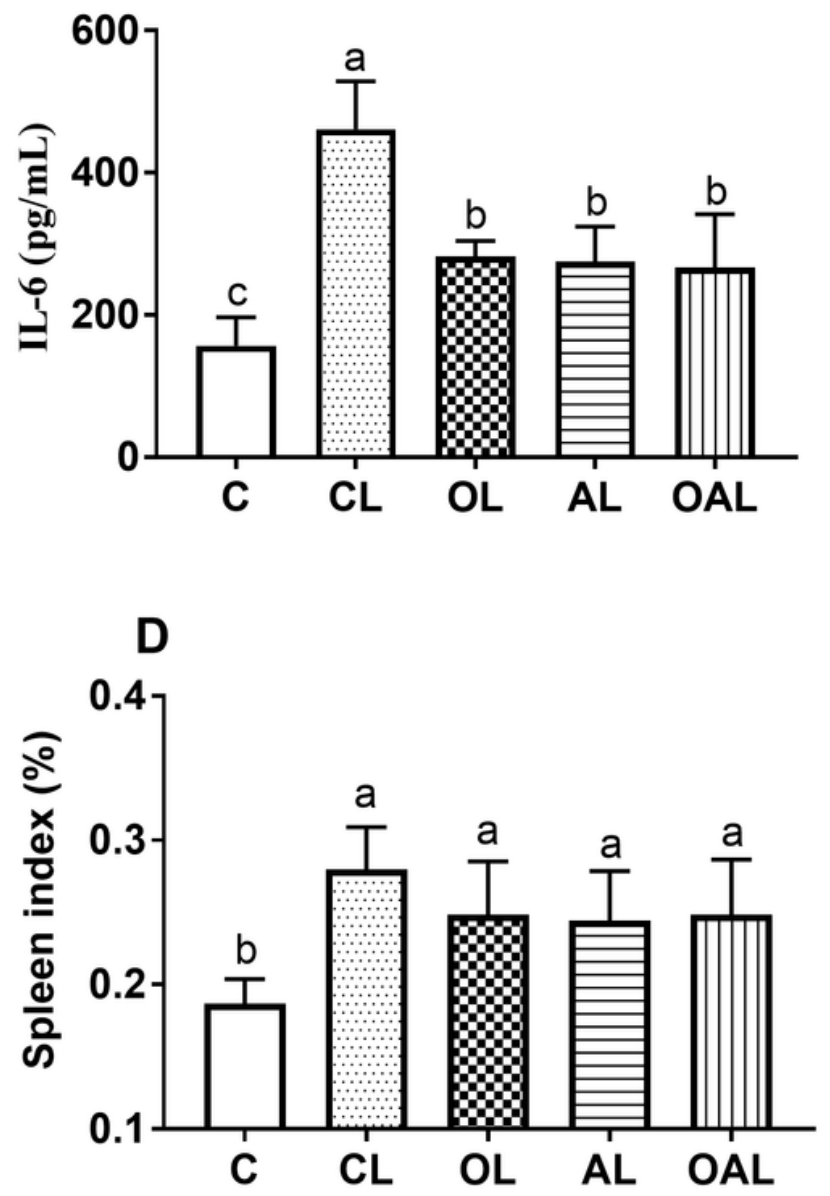

Figure 4

Serum cytokines and spleen index (iner organ weight to body weight ratio) in control piglets and those fed basal, antibiotics, olive extract, or antibiotics and olive extract diet and intraperitoneally (i.p.) injected with LPS. A-D: TNF-a, IL-6, IL-10, spleen index, respectively. Values are shown as means $\pm S D, n=6$. Labeled means with different letters significantly differ, $P<0.05$. C, control piglets fed with a basal diet; 
$\mathrm{CL}, \mathrm{AL}, \mathrm{OL}$, and OAL, piglets challenged with LPS (i.p. injection at $20 \mu \mathrm{g} / \mathrm{kg}$ body weight) after being fed with the basal diet, or the basal diet supplemented with antibiotics, olive extract, or antibiotics and olive extract, respectively, for 2 weeks. IL, interleukin; TNF-a, tumor necrosis factor-a.

A

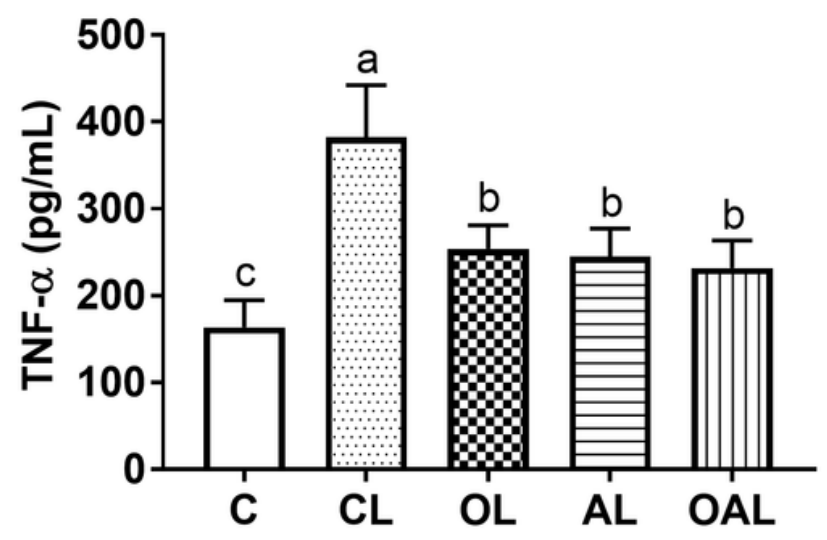

C

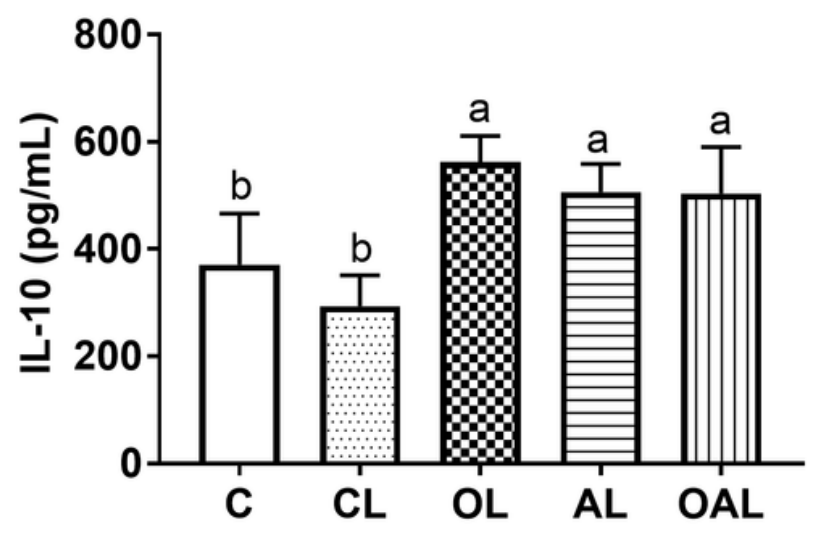

B
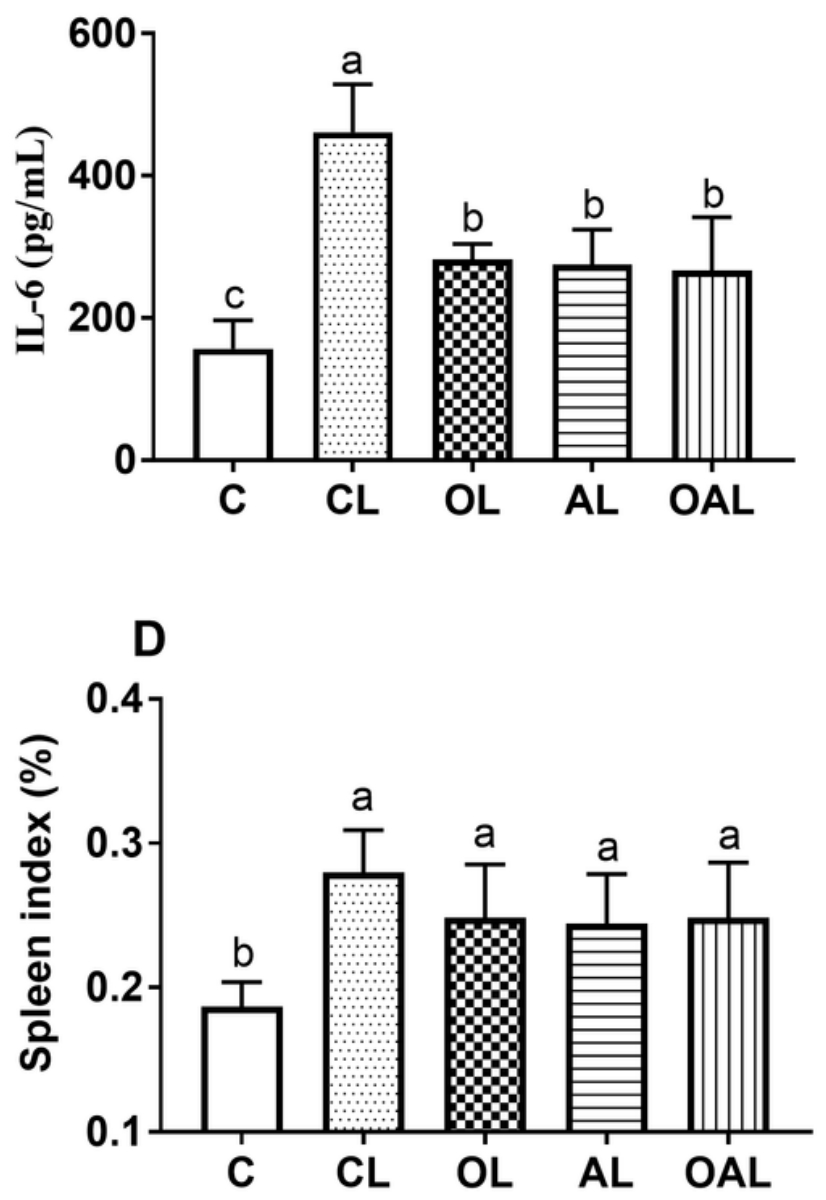

Figure 4

Serum cytokines and spleen index (iner organ weight to body weight ratio) in control piglets and those fed basal, antibiotics, olive extract, or antibiotics and olive extract diet and intraperitoneally (i.p.) injected with LPS. A-D: TNF-a, IL-6, IL-10, spleen index, respectively. Values are shown as means \pm SD, $n=6$. Labeled means with different letters significantly differ, $\mathrm{P}<0.05$. C, control piglets fed with a basal diet; $\mathrm{CL}, \mathrm{AL}, \mathrm{OL}$, and OAL, piglets challenged with LPS (i.p. injection at $20 \mu \mathrm{g} / \mathrm{kg}$ body weight) after being fed with the basal diet, or the basal diet supplemented with antibiotics, olive extract, or antibiotics and olive extract, respectively, for 2 weeks. IL, interleukin; TNF-a, tumor necrosis factor-a. 

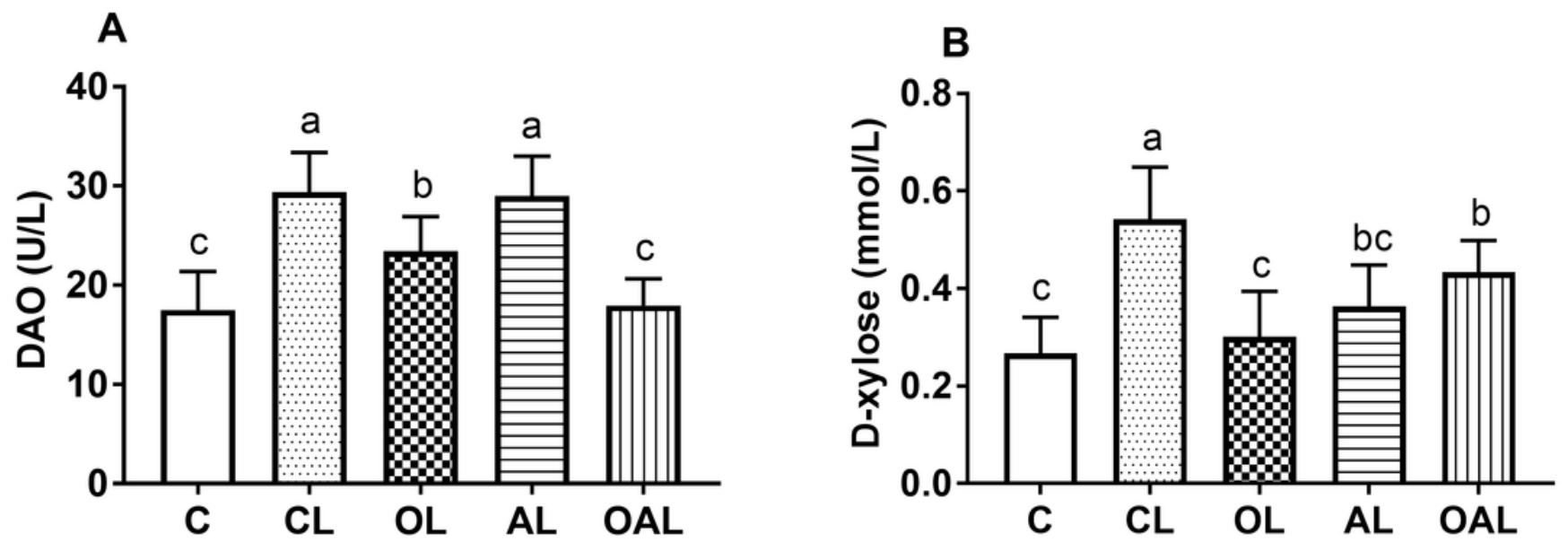

Figure 5

Serum DAO (A) and D-xylose (B) in control piglets and those fed basal, antibiotics, olive extract, or antibiotics and olive extract diet and intraperitoneally (i.p.) injected with LPS. Values are shown as means $\pm S D, n=6$. Labeled means with different letters significantly differ, $P<0.05$. C, control piglets fed with a basal diet; $\mathrm{CL}, \mathrm{AL}, \mathrm{OL}$, and OAL, piglets challenged with LPS (i.p. injection at $20 \mu \mathrm{g} / \mathrm{kg}$ body weight) after being fed with the basal diet, or the basal diet supplemented with antibiotics, olive extract, or antibiotics and olive extract, respectively, for 2 weeks. DAO, diamine oxidase.
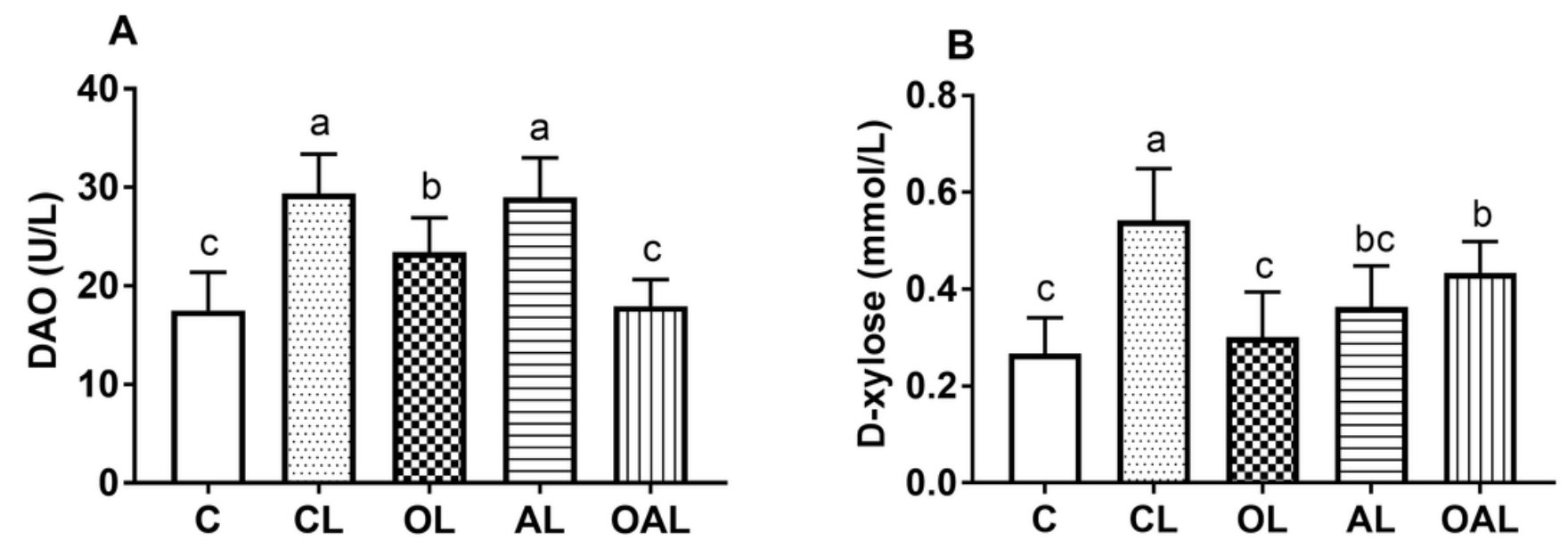

Figure 5

Serum DAO (A) and D-xylose (B) in control piglets and those fed basal, antibiotics, olive extract, or antibiotics and olive extract diet and intraperitoneally (i.p.) injected with LPS. Values are shown as means $\pm S D, n=6$. Labeled means with different letters significantly differ, $P<0.05$. C, control piglets fed with a basal diet; $\mathrm{CL}, \mathrm{AL}, \mathrm{OL}$, and OAL, piglets challenged with LPS (i.p. injection at $20 \mu \mathrm{g} / \mathrm{kg}$ body weight) after being fed with the basal diet, or the basal diet supplemented with antibiotics, olive extract, or antibiotics and olive extract, respectively, for 2 weeks. DAO, diamine oxidase. 

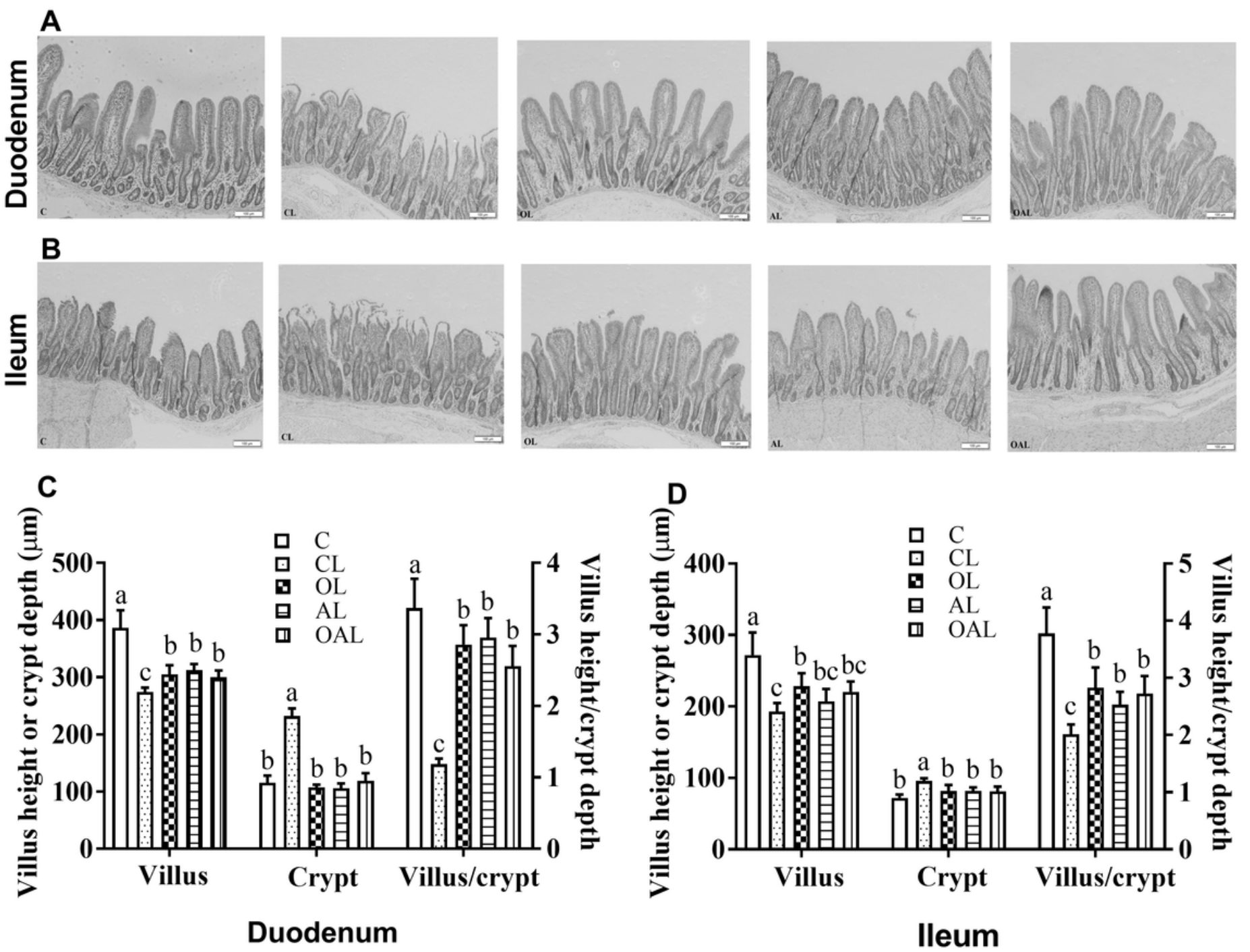

Figure 6

Histology, villus height and crypt depth of duodenum and ileum in control piglets and those fed basal, antibiotics, olive extract, or antibiotics and olive extract diet and intraperitoneally (i.p.) injected with LPS. A-B: duodenum and ileum histology, respectively. C-D: duodenum and ileum histology villus height and crypt depth, respectively. Values are shown as means $\pm S D, n=6$. Labeled means with different letters significantly differ, $\mathrm{P}<0.05$. C, control piglets fed with a basal diet; $C L, A L, O L$, and $O A L$, piglets challenged with LPS (i.p. injection at $20 \mu \mathrm{g} / \mathrm{kg}$ body weight) after being fed with the basal diet, or the basal diet supplemented with antibiotics, olive extract, or antibiotics and olive extract, respectively, for 2 weeks. 

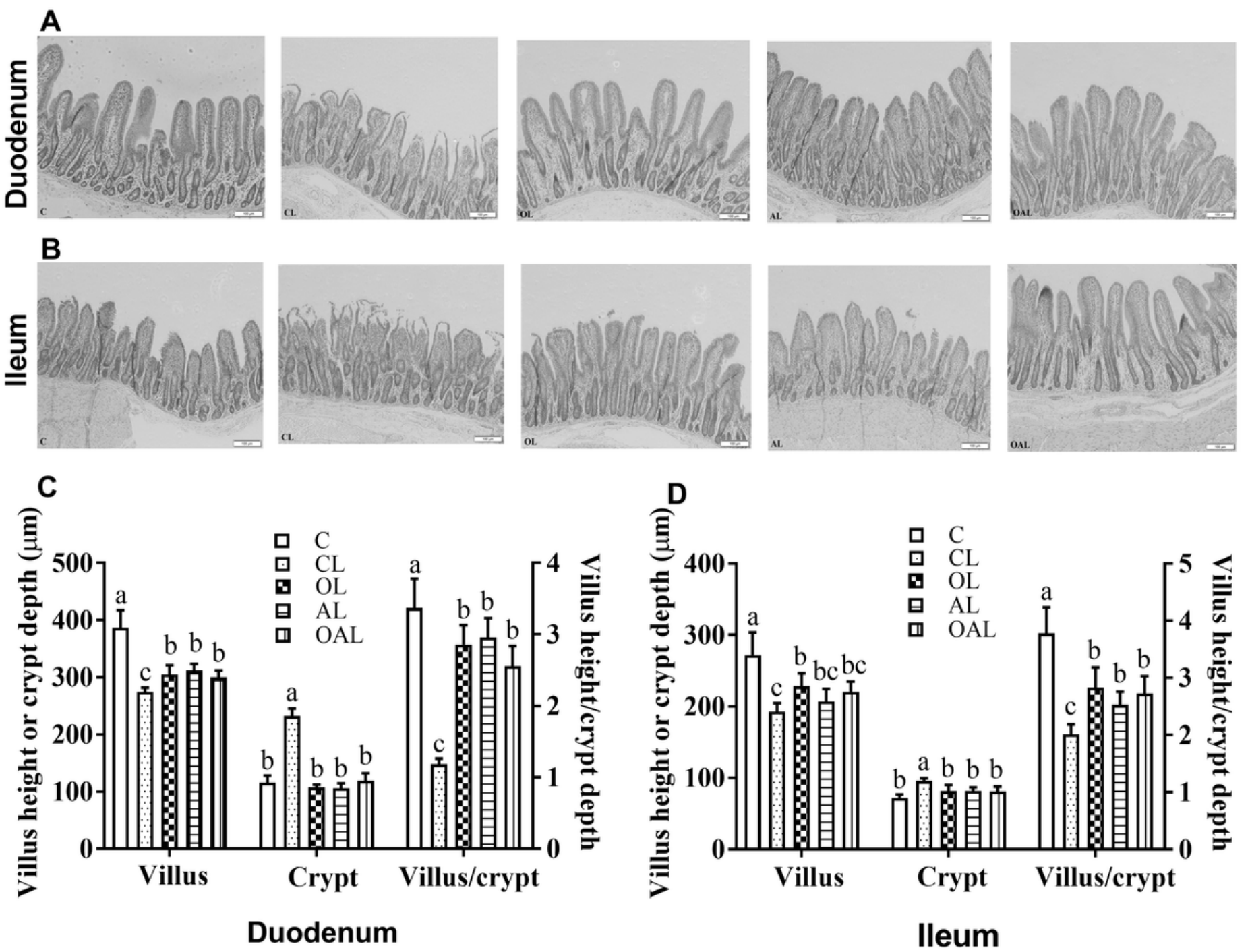

Figure 6

Histology, villus height and crypt depth of duodenum and ileum in control piglets and those fed basal, antibiotics, olive extract, or antibiotics and olive extract diet and intraperitoneally (i.p.) injected with LPS. A-B: duodenum and ileum histology, respectively. C-D: duodenum and ileum histology villus height and crypt depth, respectively. Values are shown as means $\pm S D, n=6$. Labeled means with different letters significantly differ, $\mathrm{P}<0.05$. C, control piglets fed with a basal diet; $C L, A L, O L$, and $O A L$, piglets challenged with LPS (i.p. injection at $20 \mu \mathrm{g} / \mathrm{kg}$ body weight) after being fed with the basal diet, or the basal diet supplemented with antibiotics, olive extract, or antibiotics and olive extract, respectively, for 2 weeks. 

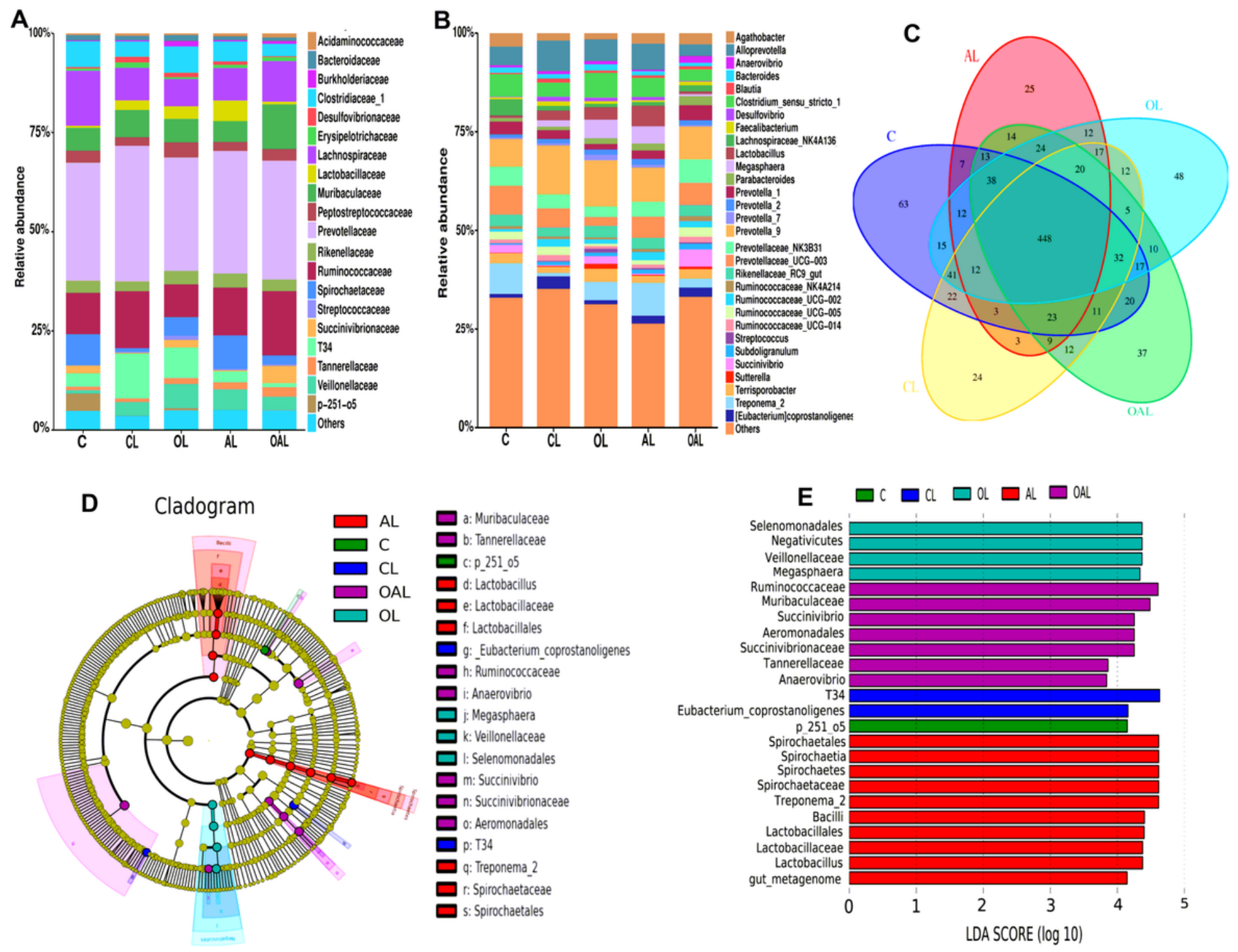

Figure 7

Microbial compositional profiles in intestine of control piglets and those fed basal, antibiotics, olive extract, or antibiotics and olive extract diet and intraperitoneally (i.p.) injected with LPS. A-E: relative abundance at the family level, relative abundance at genus levels, OTUs, bacterial taxa cladogram, LDA score, respectively. Values are shown as means $\pm S D, n=6$. Labeled means with different letters significantly differ, $P<0.05$. C, control piglets fed with a basal diet; $C L, A L, O L$, and $O A L$, piglets challenged with LPS (i.p. injection at $20 \mu \mathrm{g} / \mathrm{kg}$ body weight) after being fed with the basal diet, or the basal diet supplemented with antibiotics, olive extract, or antibiotics and olive extract, respectively, for 2 weeks. LDA, linear discriminant analysis; OTUs, operational taxonomic units. 

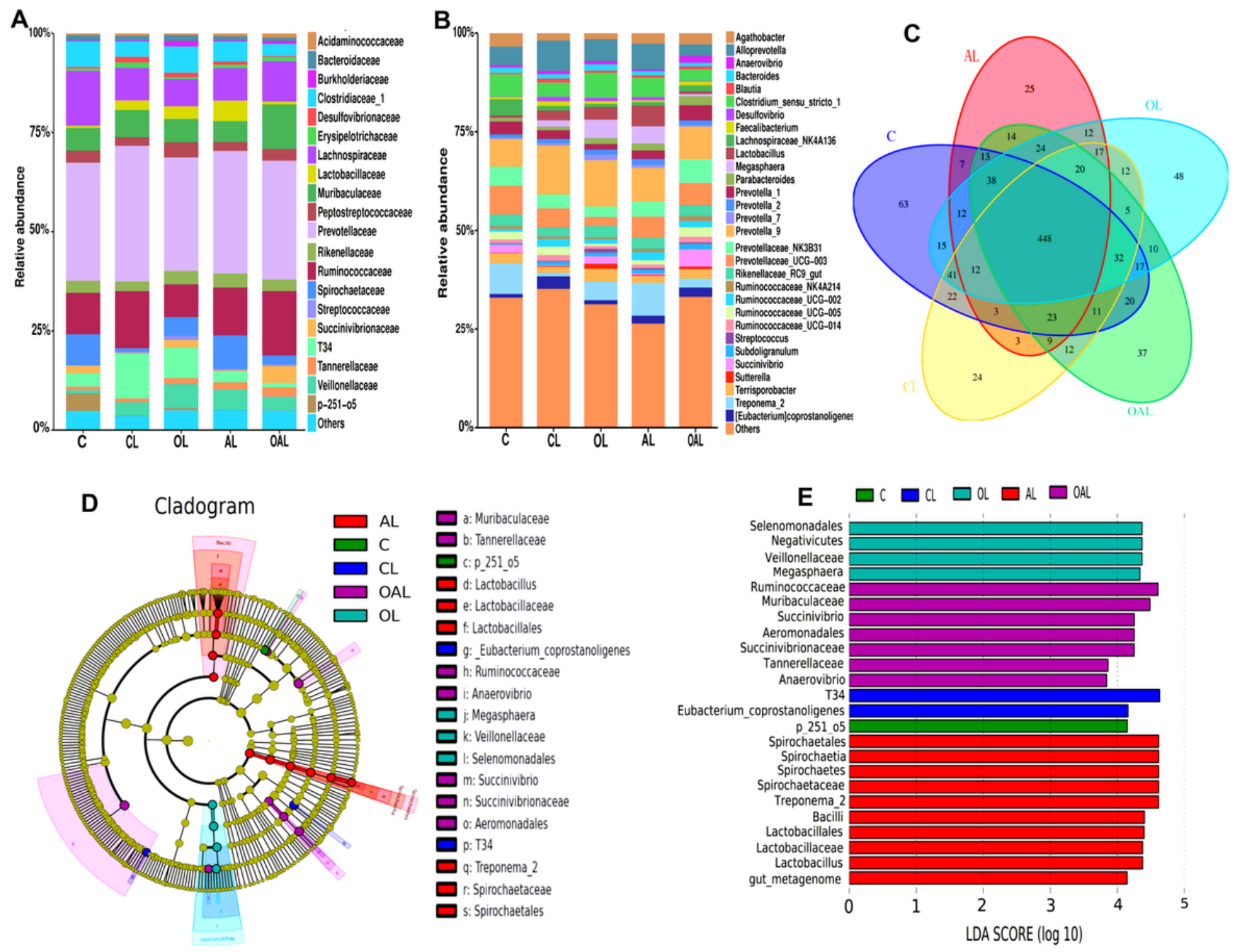

Figure 7

Microbial compositional profiles in intestine of control piglets and those fed basal, antibiotics, olive extract, or antibiotics and olive extract diet and intraperitoneally (i.p.) injected with LPS. A-E: relative abundance at the family level, relative abundance at genus levels, OTUs, bacterial taxa cladogram, LDA score, respectively. Values are shown as means $\pm S D, n=6$. Labeled means with different letters significantly differ, $P<0.05$. C, control piglets fed with a basal diet; $C L, A L, O L$, and $O A L$, piglets challenged with LPS (i.p. injection at $20 \mu \mathrm{g} / \mathrm{kg}$ body weight) after being fed with the basal diet, or the basal diet supplemented with antibiotics, olive extract, or antibiotics and olive extract, respectively, for 2 weeks. LDA, linear discriminant analysis; OTUs, operational taxonomic units. 

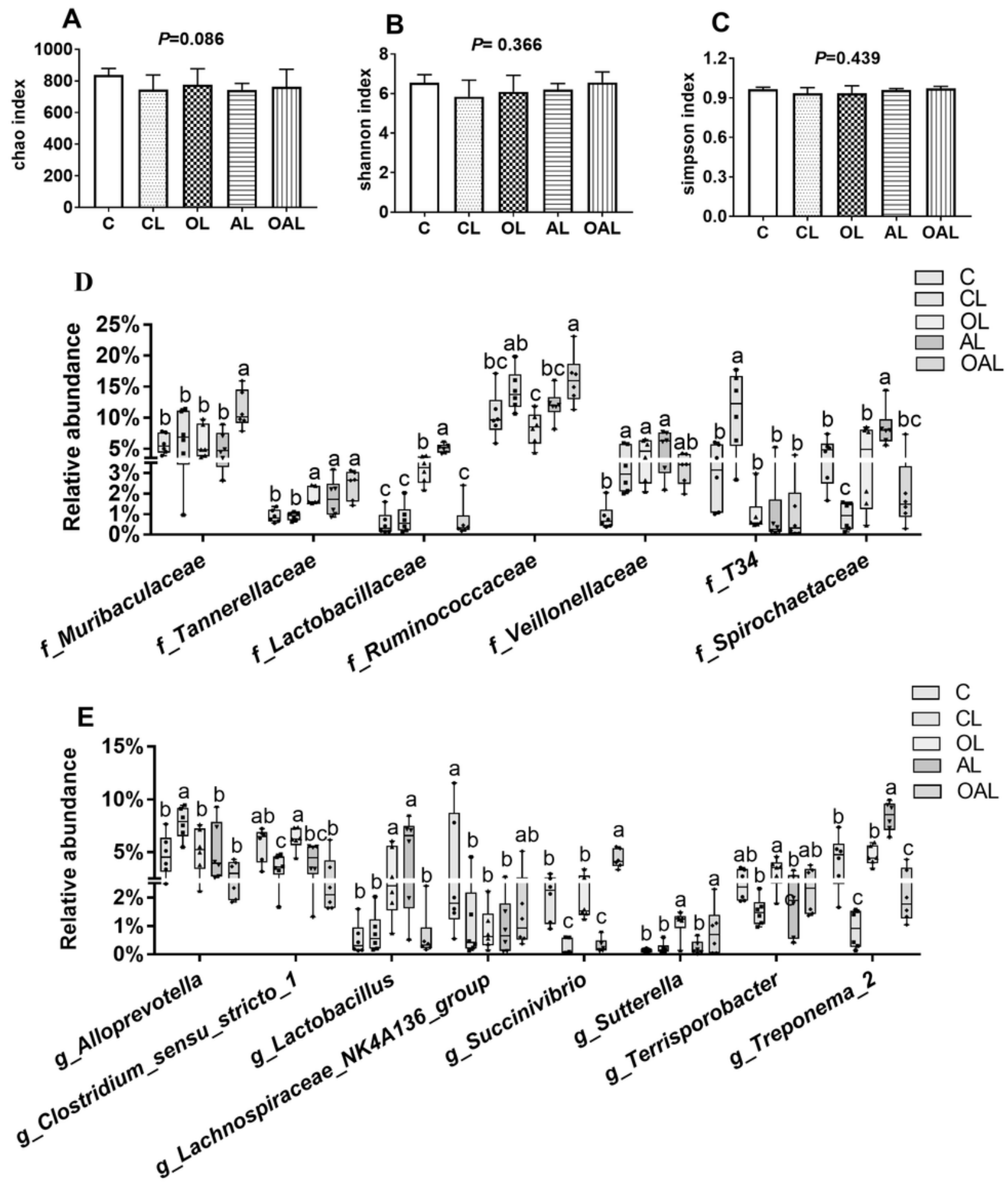

Figure 8

Microbial community diversity and relative abundance in the intestine of control piglets and those fed basal, antibiotics, olive extract, or antibiotics and olive extract diet and intraperitoneally (i.p.) injected with LPS. A-E: Chao index, Shannon index, Simpson index, relative abundance at the family level, relative abundance at genus levels, respectively. Values are shown as means $\pm S D, n=6$. Labeled means with different letters significantly differ, $\mathrm{P}<0.05$. $\mathrm{C}$, control piglets fed with a basal diet; $C L, A L, O L$, and $O A L$, 
piglets challenged with LPS (i.p. injection at $20 \mu \mathrm{g} / \mathrm{kg}$ body weight) after being fed with the basal diet, or the basal diet supplemented with antibiotics, olive extract, or antibiotics and olive extract, respectively, for 2 weeks.
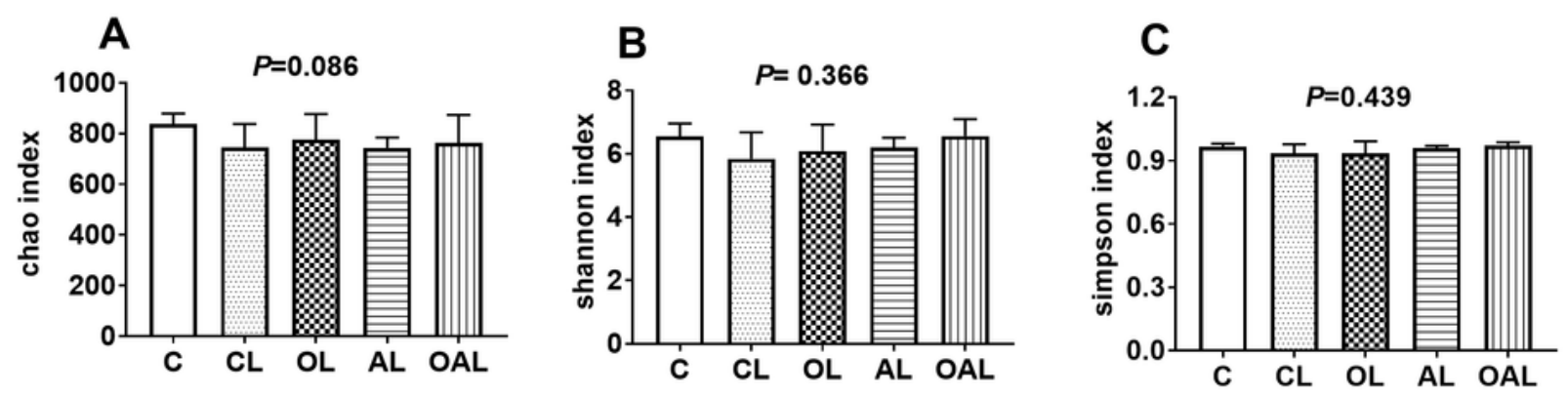

D
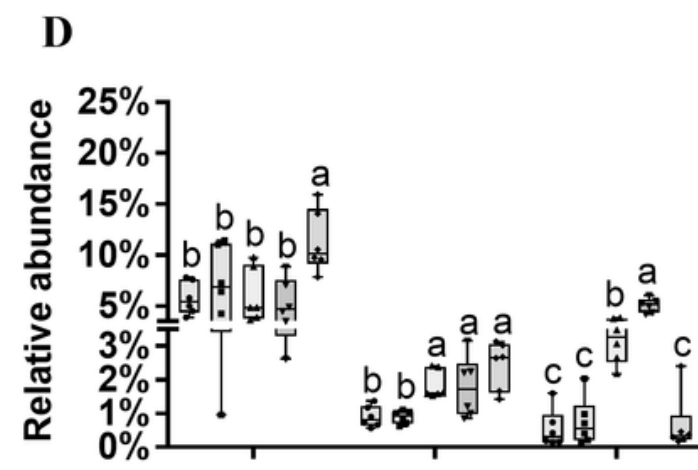
LPS. A-E: Chao index, Shannon index, Simpson index, relative abundance at the family level, relative abundance at genus levels, respectively. Values are shown as means $\pm S D, n=6$. Labeled means with different letters significantly differ, $P<0.05$. $C$, control piglets fed with a basal diet; $C L, A L, O L$, and $O A L$, piglets challenged with LPS (i.p. injection at $20 \mu \mathrm{g} / \mathrm{kg}$ body weight) after being fed with the basal diet, or the basal diet supplemented with antibiotics, olive extract, or antibiotics and olive extract, respectively, for 2 weeks. 